\title{
Comprehensive analysis of the longan transcriptome reveals distinct regulatory programs during the floral transition
}

Dengwei Jue ${ }^{1,3+}$, Xuelian Sang ${ }^{1+}$, Liqin Liu', Bo Shu', Yicheng Wang ${ }^{1}$, Chengming Liu², Yi Wang ${ }^{1}$, Jianghui $\mathrm{Xie}^{1 *}$ and Shengyou Shi ${ }^{1,3^{*}}$ (D)

\begin{abstract}
Background: Longan (Dimocarpus longan Lour.) is an important fruit tree in the subtropical regions of Southeast Asia and Australia. Among the factors affecting D. longan fruit yield, the difficulty and instability of blossoming is one of the most challenging issues. Perpetual flowering (PF) is a crucial trait for fruit trees and is directly linked to production potential. Therefore, studying the molecular regulatory mechanism of longan PF traits is crucial for understanding and solving problems related to flowering. In this study, comparative transcriptome analysis was performed using two longan cultivars that display opposite flowering phenotypes during floral induction.

Results: We obtained $853.72 \mathrm{M}$ clean reads comprising $125.08 \mathrm{~Gb}$. After comparing these data with the longan genome, 27,266 known genes and 1913 new genes were detected. Significant differences in gene expression were observed between the two genotypes, with 6150 and 6202 differentially expressed genes (DEGs) for 'SJ' and 'SX', respectively. The transcriptional landscape of floral transition at the early stage was very different in these two longan genotypes with respect to key hormones, circadian rhythm, sugar metabolism, and transcription factors. Almost all flowering-related DEGs identified are involved in photoperiod and circadian clock pathways, such as CONSTANS-like (COL), two-component response regulator-like (APRRs), gigantea (Gl), and early flowering (EFL). In addition, the leafy ( $L F Y$ ) gene, which is the central floral meristem identity gene, may inhibit PF formation in 'SJ'.

Conclusion: This study provides a platform for understanding the molecular mechanisms responsible for changes between PF and seasonal flowering (SF) longan genotypes and may benefit studies on PF trait mechanisms of evergreen fruit trees.
\end{abstract}

Keywords: Longan, Floral transition, Perpetual flowering, Comprehensive transcriptome analysis

\section{Background}

Longan (Dimocarpus longan Lour.) is an important subtropical fruit tree that is widely grown in several subtropical and tropical countries [1]. As the country of origin, China has the highest longan production in the world $[2,3]$. In general, longan varieties, such as the main cultivar 'Shixia' ('SX'), exhibit seasonal flowering (SF). Floral bud induction in $D$. longan requires favorable conditions such as a period of low temperature

\footnotetext{
* Correspondence: xiejianghui@21cn.com; ssy7299@sohu.com

${ }^{\dagger}$ Dengwei Jue and Xuelian Sang contributed equally to this work.

${ }^{1}$ Key Laboratory of Tropical Fruit Biology (Ministry of Agriculture), South

Subtropical Crops Research Institute, Chinese Academy of Tropical

Agricultural Sciences, Zhanjiang 524091, China

Full list of author information is available at the end of the article
}

(vernalization), suitable salinity and dry conditions. To obtain a stable high yield, longan flowering in the off-season is achieved through chemical treatment with potassium chlorate $\left(\mathrm{KClO}_{3}\right)[4,5]$, and region and tree variety greatly influence the induction effect. Therefore, research on the molecular regulatory mechanism of floral induction in longan is crucial for understanding and solving problems related to flowering. However, such knowledge regarding floral induction in longan is scarce because of its long generation time. The longan cultivar 'Sijimi' ('SJ') is a perpetual flowering (PF) genotype, which flowers and bears fruits throughout the year and does not require special external environmental

(c) The Author(s). 2019 Open Access This article is distributed under the terms of the Creative Commons Attribution 4.0 International License (http://creativecommons.org/licenses/by/4.0/), which permits unrestricted use, distribution, and reproduction in any medium, provided you give appropriate credit to the original author(s) and the source, provide a link to the Creative Commons license, and indicate if changes were made. The Creative Commons Public Domain Dedication waiver (http://creativecommons.org/publicdomain/zero/1.0/) applies to the data made available in this article, unless otherwise stated. 
conditions. Therefore, this cultivar is a good material for the study of longan flowering.

As an important developmental process in the plant life cycle, flowering is directly linked to production, regardless of when seeds or fruits are harvested [6], and thus flowering at an appropriate time is important for crop yield. In Arabidopsis and other model plants, the molecular mechanisms of flowering have been well established. There are at least five major flowering pathways in Arabidopsis, including photoperiod, autonomous, vernalization, gibberellin (GA), and aging pathways [7], that activate or inhibit floral transformation through a series of flower integron genes such as flowering locus $\mathrm{T}(F T)$, flowering locus $\mathrm{C}$ (FLC), and CONSTANS (CO) [8]. However, due to long generation times and complex genetic backgrounds, knowledge of the molecular genetics of flowering in perennials is scant compared to that in model plants [9]. For example, overexpression of Arabidopsis $L F Y$ in poplar (Populus spp.) resulted in early flowering in the transgenic lines [10], and flowers were observed within several months on transgenic lines overexpressing FT1 and FT2, whereas the first flowers on wild-type trees were obtained after 5-10 years [11, 12]. In addition, ectopic expression of four alternative splicing forms of Chrysanthemum FTL1 in Arabidopsis caused varying degrees of early flowering [13]. Moreover, longan FT1 and FT2 have been ectopically expressed in Arabidopsis, whereby DlFT1-and DlFT2-overexpressing lines showed early flowering and late flowering phenotypes, respectively. Interestingly, transgenic Arabidopsis lines overexpressing longan apetala1 (AP1) displayed a range of flowering time phenotypes [14]. Nonetheless, some flowering time-associated genes in Arabidopsis consistently fail to affect blossoming in trees. For example, overexpressed MADS1 and CO, LFY, AP1, and agamous-like20 (AGL20) in poplar, the transgenic lines showed very rare and early floral onset or no flowers, suggesting the presence of different flowering regulatory mechanisms in perennials [15].

Because it extends the production period, PF is a crucial trait for fruit trees [16], and the genetic control of PF has been elucidated in several model plants. For instance, flowering 1 (PEP1), an ortholog of the floral repressor FLC, controls the PF trait in Arabidopsis [17], whereas PF in diploid strawberry and rose is due to a mutation in an ortholog of the floral repressor terminal flower 1 (TFL1) $[16,18]$. Recent studies have also shown that the major FaPFRU locus, a nonortholog of TFL1, controls the PF trait in some strawberry cultivars [19, 20]. Regardless, multiyear delays in the onset of flowering and a long juvenile phase hamper studies of PF traits in perennials. Despite several studies on flowering genes in 'SJ' using RNA sequencing (RNA-Seq) analysis [4, 5], the molecular mechanism of PF traits in 'SJ' remains unknown. In this study, comparative transcriptome analysis during the floral induction process was performed using two longan cultivars ('SJ' and 'SX'). Our aim is to clarify the genetic basis for different flowering capabilities between these two cultivars. The results of this study may provide valuable information regarding the molecular regulatory mechanisms of floral induction in two longan cultivars that differ in flowering time.

\section{Methods}

\section{Plant materials}

D. longan 'SJ' and 'SX' (nine-year-old trees), which display opposite flowering phenotypes, were grown at an experimental orchard of the South Subtropical Crops Research Institute of the Chinese Academy of Tropical Agricultural Science in Zhanjiang, China $\left(110^{\circ} 16^{\prime} \mathrm{E}, 21^{\circ}\right.$ $10^{\prime} \mathrm{N}$ ). 'SX', one of the main varieties in China originating from Guangxi Province, exhibits SF traits, and floral bud induction requires a period of low temperature [21]. 'SJ', originating from China (Guangxi Province)/the Vietnam border region, exhibits PF traits, flowering and bearing fruits throughout the year under both high and low temperatures [22]. Previous molecular marker analyses have showed that 'SJ' has a close genetic relationship with longan cultivars of Guangxi Province and is clustered with Chinese cultivar groups including ' $\mathrm{SX}$ ' $[21,23]$. Three different types of 'SJ' and 'SX' apical buds were used in this study. Samples of the dormant stage (before the emergence of floral primordia) (T1) were collected on November 20, 2016; the apical bud at this stage is characterized by high hardness. Samples of floral primordia (red bud) (T2 stage) were collected on December 24, 2016; the apical bud at this stage is characterized by the appearance of red dot. Samples of the floral organ formation stage (T3) were collected on January 1, 2017; the apical bud at this stage is characterized by the appearance of the first inflorescence. Three biological replicates from three different trees were used for each sample. All samples were collected between $10 \mathrm{am}$ and $12 \mathrm{am}$, placed immediately in liquid nitrogen and stored at $-80^{\circ} \mathrm{C}$ until RNA-Seq and quantitative reverse transcription polymerase chain reaction (qRT-PCR).

\section{RNA extraction and Illumina sequencing}

Total RNA was obtained using a quick RNA Isolation Kit (Huangyueyang, Beijing, China) according to the manufacturer's instructions, and contaminant DNA was removed. The concentration and quality of the RNA were verified using an Agilent 2100 Bioanalyzer (Agilent Technologies, Palo Alto, CA, USA). Equal amounts of total RNA extracted from the three replicate plants at each flowering time comprised the cDNA library. Eighteen cDNA libraries ( 2 cultivars $\times 3$ flowering times $\times 3$ replicates) were constructed and sequenced using the Illumina $\mathrm{HiSeq}^{\mathrm{Tm}} 2000$ platform (Illumina Inc., CA, USA). Before assembly, adaptor sequences were removed 
from the raw reads. To obtain more reliable results, low-quality reads with over $50 \%$ bases were removed from each dataset. Those with quality scores of 5 or lower and/or over $10 \%$ bases unknown ( $\mathrm{N}$ bases) were also removed. High-quality clean reads from 18 samples were mapped to the longan genome database [24] using SOAPaligner/soap2 [25].

\section{Differential gene expression (DEG) analysis}

The level of unigene expression was normalized by calculating the reads per kilobase of exon model per million mapped reads (RPKM), as estimated using RSEM v1.2.15 [26]. To detect transcriptional changes in 'SJ' and 'SX' during flower induction, differential expression analysis was carried out using the DESeq R package (1.10.1) [27], which provides statistical routines for determining differential expression in digital gene expression data using a model based on negative binomial distribution. The resulting $p$ values were adjusted using the Benjamini and Hochberg approach for controlling the false discovery rate (FDR). Genes with a minimal 2-fold difference in expression ( $\mid \log _{2}$ Ratio $\left.\mid \geq 1\right)$ and an adjusted $p$-value $<0.05$ were considered differentially expressed [28]. To evaluate gene expression patterns during floral induction in 'SJ' and 'SX', expression pattern analyses were performed, and DEGs for 'SJ' and 'SX' were clustered into eight expression profiles using Short Time-series Expression Miner (STEM) version 1.3.8 [29]. The clustered profiles of DEGs with $p$-value $<0.05$ were considered significantly different from the reference set for each genotype.

To verify biological significance, all DEGs were subjected to Gene Ontology (GO) enrichment analysis using Blast2GO (version 2.8) [30] and WEGO [31]. GO terms with and adjusted $p$-value ( $p$.adjust) $\leq 0.05$ were considered significantly enriched. DEGs were mapped to terms in the Kyoto Encyclopedia of Genes and Genomes (KEGG) database using BLASTX software [32]. Pathways with a q-value $\leq 0.05$ were considered significantly enriched.

Transcription factors (TFs) were obtained from a previous study [24], and Mapman visualization [33] was performed to identify in the transcriptome data TFs that may play essential roles in regulating longan floral induction. Fragments per kilobase of the exon model per million mapped values (FPKM) were $\log _{2}$-transformed, and heat maps with hierarchical clustering were generated using the software Mev4.9.0 [34].

\section{Gene expression validation}

Thirty-eight DEGs were selected to confirm the transcriptomic data through qRT-PCR analysis. Gene-specific primers were designed using Primer 3 software (Additional file 1). The three independent biological replicates for three flowering development times were mixed as T1, T2, and T3. First-strand cDNA was generated from purified total RNA using a PrimeScript ${ }^{\mathrm{TM}} \mathrm{RT}$ reagent kit (Takara, Japan). Longan actin (Dlo_028674), which was used in our previous study, was selected as the reference gene [35]. qRT-PCR was conducted using the LightCycler ${ }^{\circ}$ 480 Real-Time PCR System (Roche, Germany) and SYBR Green II PCR Master Mix (Takara, Japan). The amplification program was as follows: $95^{\circ} \mathrm{C}$ for $5 \mathrm{~min}$, followed by 40 cycles of $95^{\circ} \mathrm{C}$ for $15 \mathrm{~s}$ and $60^{\circ} \mathrm{C}$ for $1 \mathrm{~min}$. Each reaction was performed in triplicate. The relative expression levels of the candidate genes were calculated using the $2^{-\Delta \Delta \mathrm{Ct}}$ method [35]. Pearson's correlation values between the RNA-Seq and qRT-PCR data of selected genes were calculated using "cor.test" in R version 3.3.

\section{Results}

Transcriptome assembly of sequencing reads

To identify genetic differences that may contribute to the PF traits of 'SJ' at the transcriptional level, comparative transcript profiling of 'SX' and 'SJ' flower buds from three different developmental stages was performed using RNA-Seq. The Illumina sequence data were deposited in the National Center for Biotechnology Information (NCBI) Sequence Read Archive (SRS2241241SRS2241258). After filtering adaptor sequences and removing low-quality tags, $853.72 \mathrm{M}$ clean reads were obtained, with 41,542,306-57,566,164 clean reads generated from the 18 samples and $125.08 \mathrm{~Gb}$ of sequence. The Q30 percentage (sequences with a sequencing error rate lower than $0.1 \%$ ) was greater than $93 \%$, and the average GC content was $44.70 \%$. Among the total clean reads, approximately 32,322,773 (77.81\%)$47,947,255$ (83.29\%) matched perfectly to the longan genome (Additional file 2) [24]. After merging these data, 27,266 (69.41\%) known genes and 1913 new genes were identified (Additional file 3). These results indicate that the sequencing quality was sufficient for further analyses.

\section{Identification of DEGs between 'SJ' and 'SX' during floral induction}

DEGs were first identified through comparisons of the RPKM values for each gene of 'SJ' and 'SX' at different floral induction stages (SXT1-vs-SJT1, SXT2-vs-SJT2, and SXT3-vs-SJT3) with $\mid \log _{2}$ Ratio $\mid \geq 1$ as the threshold of expression fold and FDR $\leq 0.05$. A total of 9714 DEGs were detected in three pairwise stage comparisons: 6857 DEGs in T1 (SXT1-vs-SJT1), 3878 DEGs in T2 (SXT2-vs-SJT2) and 4919 DEGs in T3 (SXT3-vs-SJT3) (Fig. 1b and Additional file 4). Among the three pairwise stage comparisons, 1833 DEGs were common, indicating genetic differences between the two genotypes. GO and KEGG classifications were then performed to determine 

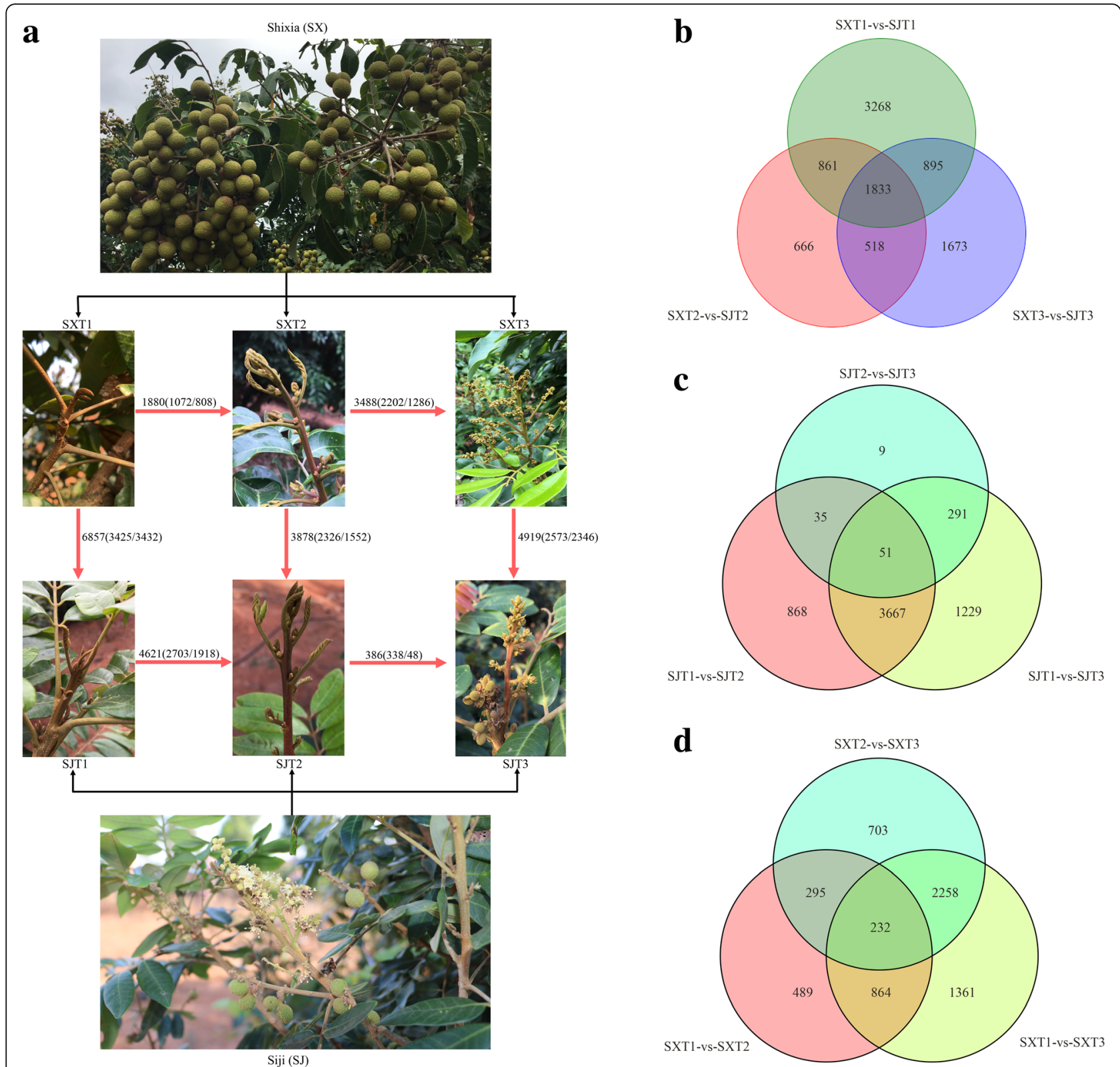

Fig. 1 Different flowering phenotypes and number of differentially expressed genes during floral induction in 'SJ' and 'SX' longan. a Different flowering traits of 'SJ' and 'SX'. 'SJ' longan blossoms continuously; both terminal and axillary shoots can differentiate into inflorescences, and flowers and fruits can be observed at the same time on one tree. $\mathrm{T} 1$ represents the dormant stage (before the emergence of floral primordia), $\mathrm{T} 2$ represents the emergence of floral primordium stage, and T3 represents the floral organ formation stage. The red arrows represent comparisons conducted in quantitative analyses. The numbers by the arrows denote the number differentially expressed genes for the specified comparison. $\mathbf{b}$ Venn diagram showing the number of DEGs between ' $S J$ ' and 'SX' longan during the floral induction process. $\mathbf{c}$ Venn diagram showing the number of DEGs during floral induction in 'SJ'. $\mathbf{d}$ Venn diagram showing the number of DEGs during floral induction in 'SX'

the functional significance of the transcriptional changes in each comparison. The significantly enriched GO terms (p.adjust $<0.05$ ) are shown in Additional file 5 . Regarding DEGs between 'SJ' and 'SX' at the dormant stage (T1), GO terms related to "cytoskeletal" and "microtubule" in the cellular component category, "microtubule-based process" and "oxidation-reduction process" in the biological process category, and those related to "binding" and "catalytic activity" in the molecular function category were significantly enriched. In the SXT2-vs-SJT2 comparison, only five significantly enriched GO terms in molecular function, including "kinase activity", "phosphotransferase activity", "chromatin binding", "actin binding", and "hexokinase activity", were found. Regarding the SXT3-vs-SJT3 comparison, GO terms related to "sequence-specific DNA binding 
transcription factor activity", "nucleic acid" and "oxidoreductase activity" in molecular function and those associated with "regulation of biological process" in biological process were significantly enriched.

In the SXT1-vs-SJT1, SXT2-vs-SJT2, and SXT3-vs-SJT3 comparisons, 813, 167, and 402 DEGs were mapped to 118, 72, and 98 KEGG pathways, respectively. Ten pathways were significantly enriched the SXT1-vs-SJT1 comparison (Additional file 6), including "starch and sucrose metabolism" (corrected $q$-value $=9.64 \times 10^{-3}, 51$ genes) and "plant hormone signal transduction" (corrected $q$-value $=1.73 \times 10^{-2}$, 106 genes). For the DEG comparison between 'ST' and 'SX' at T2, pathways of "plant-pathogen interaction" (corrected $q$-value $=1.22 \times 10^{-4}, 54$ genes), "plant hormone signal transduction" (corrected $q$-value $=1.31 \times 10^{-2}, 31$ genes), and "circadian rhythm-plant" (corrected $q$-value $=2.26 \times 10^{-2}, 8$ genes) were significantly enriched. Finally, "plant hormone signal transduction" (corrected $q$-value $=8.37 \times 10^{-4}$, 67 genes) and "plant-pathogen interaction" (corrected $q$-value $=8.47 \times 10^{-3}, 98$ genes) were significantly enriched DEGs between 'SJ' and 'SX' at the T3 stage.

The results of GO and KEGG enrichment analyses suggested that the DEGs between 'SJ' and 'SX' at the dormant stage (T1) are mainly related to genes involved in plant basal metabolic processes; DEGs between 'SJ' and 'SX' at T2 and T3 are mainly involved in signal transduction and environmental adaptation. Interestingly, genes involved in circadian rhythm were only significantly enriched in the T2 stage, the emergence of the floral primordia, which is the signal for flower initiation.

\section{Quantitative transcriptomic changes during flower induction in ' $\mathrm{SJ}$ ' and ' $\mathrm{S} \mathrm{X}$ '}

Gene expression over the developmental course of flower induction was comparatively examined (T1-vsT2, T2-vs-T3, and T1-vs-T3), revealing that 6150 and 6202 DEGs were significantly expressed in 'SJ' and 'SX', respectively (Fig. 1c, Additional file 7 and Additional file 8). Moreover, the distribution of changes between adjacent developmental stages was biased toward early floral induction in 'SJ', and 4621 (T1-vs-T2) and 386 (T2-vs-T3) DEGs were found (Fig. 1c), whereas the distribution of changes in 'SX' was biased toward the late floral induction stage, with 1880 (T1-vs-T2) and 3488 (T2-vs-T3) DEGs found. In addition, 5238 and 4715 DEGs were found in SJT1-vs-SJT3 and SXT1-vs-SXT3, respectively (Fig. 1d).

To evaluate the expression profiles, trend analyses were performed using STEM software, and the DEGs in 'SJ' and 'SX' clustered into 8 profiles. In 'S)', 4 profiles had a significance of $p<0.05$ (J0, J1, J6, and J7 with 400, 1878, 2796, and 448 DEGs, respectively) (Fig. 2a). GO functions and KEGG pathway enrichment in each main profile were also analyzed (Additional files 9 and 10). Profile 0 of 'SJ' had an overrepresentation of genes from processes associated with "photosynthesis", "oxidation-reduction", and "ATP/ADP binding"; significant pathways in this profile were "AGE-RAGE signaling pathway in diabetic complications" ( $q$-value $=1.30 \times 10^{-2}, 4$ genes), "photosynthesis-antenna proteins" ( $q$-value $=1.39 \times 10^{-2}, 3$ genes $)$, and "plant-pathogen interaction" ( $q$-value $=3.40 \times 10^{-2}, 31$ genes). Expression of the DEGs in profile 0 was decreased during the entire stage. Profile 1 showed more significantly enriched GO terms than did profile 0 , and most of these 140 significantly enriched GO terms are related to "binging", "catalytic activity", "stress response", and "regulation of biological process". Moreover, genes involved in "limonene and pinene degradation" ( $q$-value $=2.37 \times 10^{-14}, 46$ genes), "stilbenoid, diarylheptanoid and gingerol biosynthesis" ( $q$-value $=5.52 \times 10^{-14}, 54$ genes), "plant-pathogen interaction" ( $q$-value $=2.87 \times 10^{-12}, 156$ genes), "benzoxazinoid biosynthesis" ( $q$-value $=7.32 \times 10^{-4}, 10$ genes $)$, and "plant hormone signal transduction" ( $q$-value $=1.56 \times 10^{-2}, 73$ genes $)$ were significantly enriched. Gene expression decreased through T1 to T2 and remained low during the subsequent stage. In profile 6 , there were 96 significantly enriched GO terms, with most DEGs being involved in "cellular biosynthetic", "macromolecule biosynthetic", "intracellular component biosynthetic" and other processes associated with cell proliferation and differentiation. The significantly enriched pathways of this profile were found to be "starch and sucrose metabolism" ( $q$-value $=3.35 \times 10^{-7}, 58$ genes $)$, "ascorbate and aldarate metabolism" ( $q$-value $=4.81 \times 10^{-7}, 25$ genes $)$, "phenylpropanoid biosynthesis" ( $q$-value $=2.33 \times 10^{-6}$, 54 genes), "pentose and glucuronate interconversions" $\left(q\right.$-value $=2.33 \times 10^{-6}, 34$ genes $)$, "plant hormone signal transduction" ( $q$-value $=5.88 \times 10^{-6}, 107$ genes $)$, "DNA replication" ( $q$-value $=1.10 \times 10^{-5}, 18$ genes $)$, "carotenoid biosynthesis" ( $q$-value $=2.10 \times 10^{-4}, 28$ genes), and "flavonoid biosynthesis" ( $q$-value $=2.05 \times 10^{-2}, 23$ genes). The expression level of these genes in profile 6 peaked at T2 and remained high during T2 to T3. However, only two GO terms ("sequence-specific DNA binding transcription factor activity" and "nucleic acid binding transcription factor activity") and one pathway ("alpha-linolenic acid metabolism") were significantly enriched in profile 7 , and gene expression in this profile increased during the entire stage.

In 'SX', 6202 DEGs clustered into 8 profiles. We also found 4 profiles with a significant $q$-value $<0.05$ (Fig. 2b), with profiles X0, X3, X4, and X7 containing 764, 110, 1613 , and 881 DEGs, respectively. Compared to 'SJ', profiles 3 and 4, in which the level of gene expression changed at the T2 to T3 transition, were significantly clustered in 'SX'. Most of the DEGs involving "catalytic activity", "molecular function regulator", "regulation of 


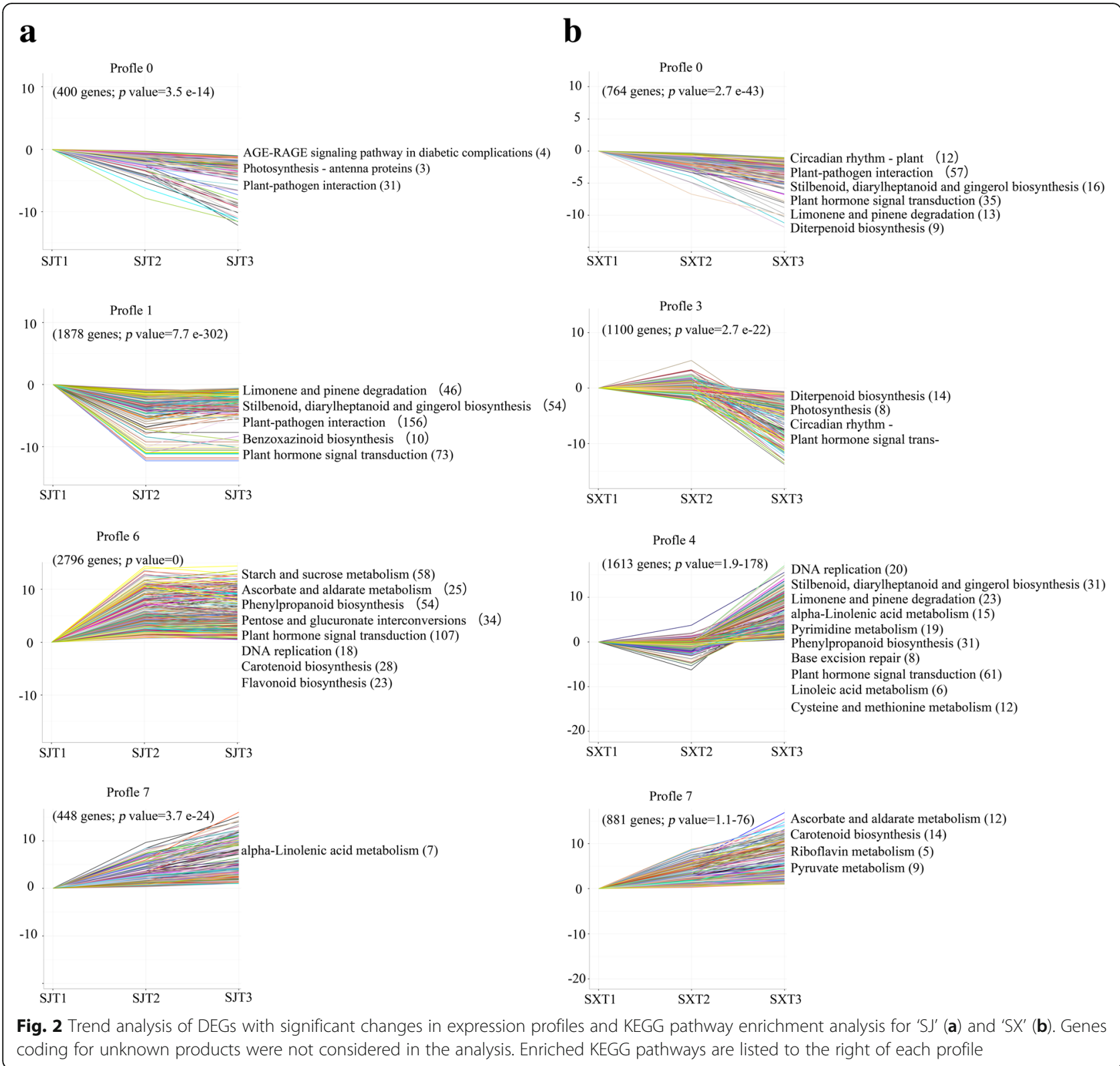

biological process", "organ development", "immune response" and other processes involved in cell proliferation and differentiation were significantly enriched in profile 0. Moreover, pathways of "circadian rhythm-plant" ( $q$-value $=4.45 \times 10^{-4}, 12$ genes), "plant-pathogen interaction" $\left(q\right.$-value $=3.37 \times 10^{-3}, 57$ genes $)$, "stilbenoid, diarylheptanoid and gingerol biosynthesis" ( $q$-value $=9.26 \times 10^{-3}, 16$ genes), "plant hormone signal transduction" ( $q$-value $=9.26 \times 10^{-3}, 35$ genes), "limonene and pinene degradation" ( $q$-value $=$ $9.26 \times 10^{-3}, 13$ genes), and "diterpenoid biosynthesis" ( $q$-value $=9.26 \times 10^{-3}, 9$ genes $)$ were overrepresented. DEGs involved in "photosynthesis" and "regulation of biosynthetic process" were significantly enriched in profile 3 , as were pathways of "diterpenoid biosynthesis" $\left(q\right.$-value $=3.37 \times 10^{-5}, 14$ genes), "photosynthesis" ( $q$-value $=2.26 \times 10^{-3}, 8$ genes), "circadian rhythm" ( $q$-value $=2.26 \times 10^{-3}, 11$ genes $)$ and "plant hormone signal transduction" ( $q$-value $=5.62 \times 10^{-3}, 39$ genes); gene expression remained at a high level at T1 to T2 and decreased during the subsequent stage. DEGs in profile 4 remained at a low level from $\mathrm{T} 1$ to $\mathrm{T} 2$ and increased during the subsequent stage. Overrepresentation of "DNA replication" ( $q$-value $=3.77 \times 10^{-10}, 20$ genes), "stilbenoid, diarylheptanoid and gingerol biosynthesis" ( $q$-value $=3.74 \times 10^{-4}, 31$ genes), "limonene and pinene degradation" ( $q$-value $=2.55 \times 10^{-3}, 23$ genes), "alpha-linolenic acid metabolism" ( $q$-value $=3.84 \times 10^{-3}$, 15 genes), "pyrimidine metabolism" ( $q$-value $=3.84 \times 10^{-3}$, 19 genes), "phenylpropanoid biosynthesis" ( $q$-value $=$ 
$3.91 \times 10^{-3}, 31$ genes), "base excision repair" ( $q$-value $=$ $1.34 \times 10^{-2}, 8$ genes), "plant hormone signal transduction" ( $q$-value $=1.48 \times 10^{-2}, 61$ genes), "linoleic acid metabolism" $\left(q\right.$-value $=1.66 \times 10^{-2}, 6$ genes $)$, and "cysteine and methionine metabolism" ( $q$-value $=2.78 \times 10^{-2}, 12$ genes $)$ were enriched in this profile. In turn, profile 7 contained more DEGs significantly enriched in "catalytic activity", "single-organism process" and cell wall association function categories, and significantly enriched pathways were "carotenoid biosynthesis" ( $q$-value $=5.17 \times 10^{-4}, 12$ genes), "ascorbate and aldarate metabolism" ( $q$-value $=3.39 \times 10^{-3}$, 14 genes), "pyruvate metabolism" ( $q$-value $=2.04 \times 10^{-2}, 5$ genes), and "riboflavin metabolism" ( $q$-value $=2.58 \times 10^{-2}$, 9 genes) (Tables S8 and S9).

Compared to 'SX', gene regulation at the transcriptional level in 'SJ' was more active with regard to the period of dormancy release (T1 to T2 transition), whereas the major transcriptional regulation of ' $\mathrm{SX}$ ' occurred at the period of floral organ formation (T2 to T3 transition). DEGs in starch and sucrose metabolism pathways were only significantly enriched in profile 6 of 'SJ', with increased expression at T1 to T2 for beta-glucosidase 40 (Dlo_026115.1), sucrose synthase (Dlo_019926.1), and endoglucanase 17 (Dlo_023528.1) (Additional file 7). In addition, DEGs associated with plant hormone signal transduction were significantly clustered in profiles 1 and 6 in 'SJ', which were up- or downregulated, respectively, during T1 to T2. Although some genes clustered in 'SX' profiles 3 and 4, they were upregulated or downregulated during the $\mathrm{T} 2$ to T3 transition, including DELLA protein GAI (Dlo_019465.1), AUX_IAA domain-containing protein (Dlo_021779.1), and auxin-induced protein 22C-like (Dlo_004907.3) (Additional file 8). Furthermore, the pathway related to photosynthesis clustered in different profiles in 'SJ' and 'SX', and the circadian rhythm pathway was only significantly enriched in profile 3 of 'SX'. The results indicate that these pathways may play crucial roles in the formation of different flowering traits between the two genotypes.

\section{DEGs related to starch and sucrose metabolism}

As the starch and sucrose metabolism pathway was only significantly enriched in profile 6 in 'SJ' and the SXT1-vs-SJT1 comparison, the 97 DEGs (Additional file 11) in this pathway were further analyzed. Compared to 'SX', 33 genes were significantly upregulated during the $\mathrm{T} 1$ to $\mathrm{T} 2$ transition in ' $\mathrm{ST}$ ', including pectinesterase, galacturan 1,4-alpha-galacturonidase, UDP-glucuronate 4-epimerase, 1,4-beta-D-xylan synthase, glgC, beta-amylase, sucrose synthase (Dlo_019926.1), beta-glucosidase, fructokinase, and endoglucanase (Fig. 3a). Moreover, 27 genes were significantly downregulated, such as GAUT (Dlo_016686.1), TPS (trehalose 6-phosphate synthase), starch synthase, PYG, alpha-glucosidase, sucrose-phosphate synthase, HK (hexokinase), and pgm (phosphoglucomutase). However, only 4 genes (1,4-beta-D-xylan synthase, Dlo_028920.1; glucose-1-phosphate adenylyltransferase, Dlo_020033.1; sucrose-phosphate synthase, Dlo_018802.4; UGDH, Dlo_007167.1) and 3 genes (polygalacturonase, Dlo_020054.1 and dlo_ 037740.1; beta-amylase, dlo_038617.1) were significantly upregulated or downregulated at the same stage in 'SX'. There were 13 and 2 common genes significantly upregulated or downregulated, respectively, in these two cultivars. No genes in 'ST' were significantly up- or downregulated during the T2 to T3 transition. Although 23 and 9 genes were significantly upregulated and downregulated at the same stage in 'SX', 4 genes common to these two cultivars were significantly upregulated.

\section{Differential gene expression in hormone signaling pathways}

The plant hormone signal transduction pathway was found to be enriched in three comparisons between ' $\mathrm{SJ}$ ' and 'SX' and in different profiles in the two cultivars during floral induction. To identify key DEGs regulating PF traits, the DEGs involved in hormone signaling were further analyzed using Mapman (Fig. 4).

In this study, 237 DEGs related to hormone signaling pathways were identified (Additional file 12). Compared to 'SX', DEGs related to hormone signaling pathways in 'S' were predominantly enriched in the early flower induction stage, with 89 and 28 DEGs displaying significant upregulation or downregulation, respectively, through $\mathrm{T} 1$ to $\mathrm{T} 2$. Among these 117 DEGs, 16 associated with the auxin pathway, including AUX1 (Dlo_003834.1), five auxin-responsive genes, two indole-3-acetic acid-amido synthetase (GH3) genes, and six SAUR family genes, showed upregulation, and one SAUR32-like gene (Dlo_023207.1) and one auxin-responsive gene (Dlo_021779.1) showed downregulation; 16 genes related to the CK pathway were upregulated, and 3 genes were downregulated, including $A H K$ and $A R R-A$. Moreover, 11 genes related to the GA pathway were upregulated, including 7 gibberellin receptor GID1 and 4 DELLA genes, and 3 GID1 and 2 DELLA genes were downregulated. Two $P P 2 C$ and $2 P Y L$ genes associated with the ABA pathway were upregulated, and 2 PP2C, 1 SRK2, and $1 A B F$ were downregulated. In addition, 33 and 7 DEGs involved in the brassinosteroid signaling pathway exhibited upregulation or downregulation, including BRI1, BSK, BRZ1/2 and CYCD3; one CTR1 (Dlo_013291.1) and one ERF1 (Dlo_022309.1) related to the ET pathway showed upregulation, and one EIN3 (Dlo_030832.1) and one ERF1 (Dlo_008317.1) displayed downregulation. Eight and two DEGs related to the JA pathway were upregulated or downregulated, respectively, during $\mathrm{T} 1$ to $\mathrm{T} 2$. Three TGA TFs related to the SA pathway displayed downregulation in 'SJ' during $\mathrm{T} 1$ to $\mathrm{T} 2$. In 'SJ', only AUX1 

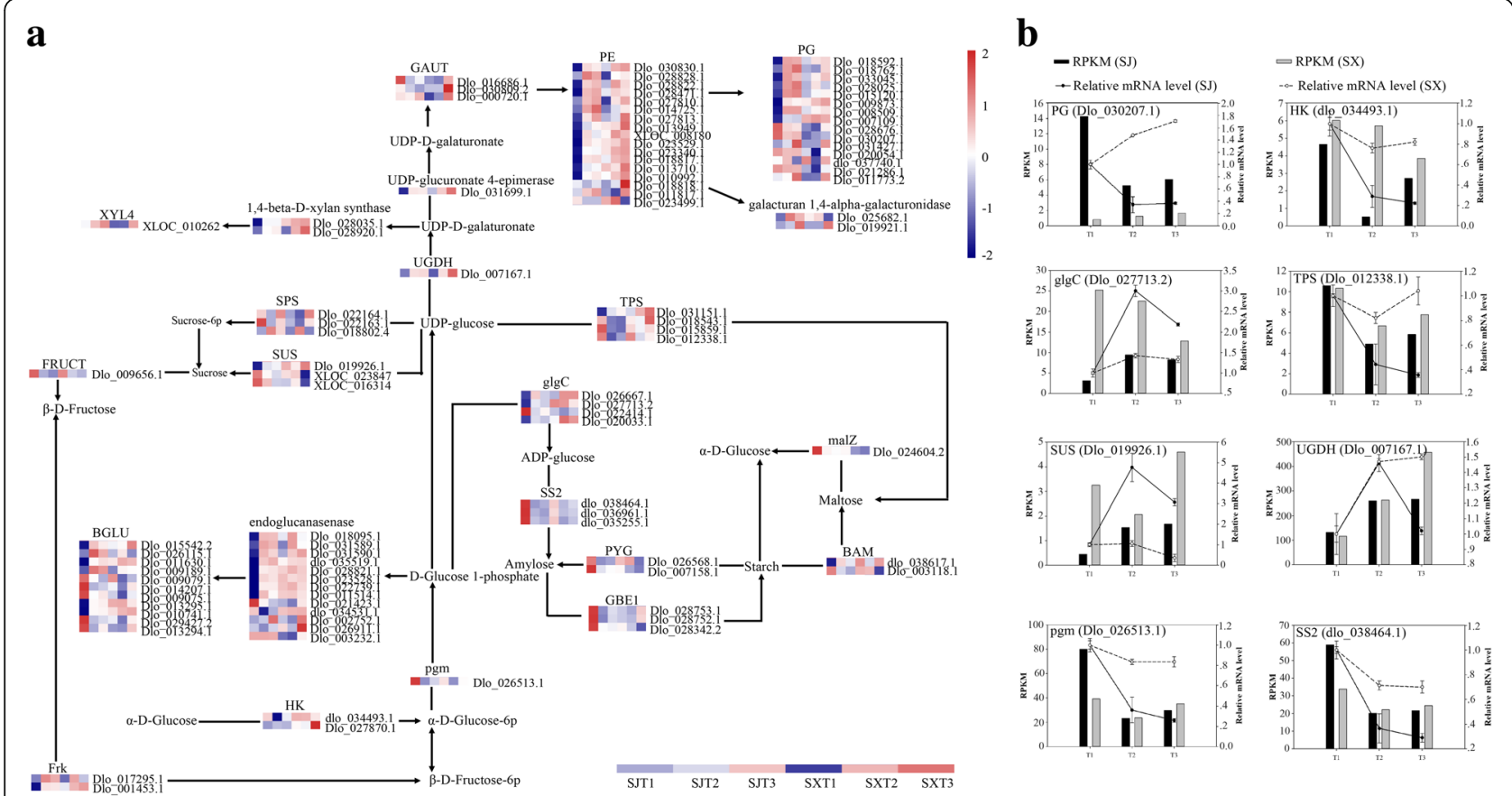

Fig. 3 Expression profiles of sugar-related genes and qRT-PCR identification of sugar-related gene expression levels in flower buds during the floral induction process in 'SJ' and 'SX' longan. a Heat map of the comparative expression levels of sugar-related genes. Data for gene expression levels were normalized by the Z-score. Red and blue indicate up- and downregulated genes, respectively. $\mathbf{b}$ qRT-PCR identification of sugarrelated gene expression levels in buds. The bar and line graphs are derived from RNA-Seq and qRT-PCR data, respectively. Values are the means of three replicates \pm SE

(XLOC_010561) and PIF3 (Dlo_027777) were upregulated or downregulated during T2 to T3. Compared to 'SJ', most DEGs related to hormone biosynthesis and signaling pathways in 'SX' were enriched in the late floral induction stage: 43 DEGs were upregulated or downregulated during T1 to T2. In contrast, 92 DEGs displayed upregulation or downregulation in 'SX' through T2 to T3 compared to 'SJ'. Among these 43 DEGs, 22 displayed significant upregulation, including one IAA gene (Dlo_025130.1), three SRURs and two $G H 3$ genes of the Aux-mediated signaling pathway, 5 GID1 and two DELLA genes involved in the GA-mediated signaling pathway, one $A H K$ gene (Dlo_004406) related to the CK pathway, and one PYL, three BRI1 and one MYC2 (Dlo_003448.1) involved in the ABA, brassinosteroid and JA-mediated signaling pathways, respectively. Twenty-one DEGs were significantly downregulated during $\mathrm{T} 1$ to $\mathrm{T} 2$ compared to 'SJ', including an auxin influx carrier family protein and ARF involved in the Aux-mediated signaling pathway, two-component response regulator ARR-A and ARR-B family proteins related to the CK pathway, and GID1, DELLA, ABF and BRI1 involved in the GA, ABA, and brassinosteroid-mediated signaling pathways. Among the 92 DEGs significantly upregulated or downregulated through T2 to T3 in 'SX', 51 were upregulated and 37 downregulated. These genes are related to auxin, GA, CK, ABA, ET and JA-mediated signaling pathways; the SA pathway was not represented in this case.

\section{Identification of flowering-related DEGs during flower induction}

A hierarchical heat map was constructed to comparatively analyze the 39 flowering-related genes (Additional file 13) identified in this study (Fig. 5). Among these 39 DEGs, in 'SJ', 6 were only significantly upregulated during T1 to T2, including three CONSTANS-like family genes, GAI (Dlo_019465.1) and two TCP genes, and seven were only significantly downregulated during $\mathrm{T} 1$ to $\mathrm{T} 2$, including an APRR family gene, EFL (Dlo_027544.1) and GI (Dlo_024864.1). In contrast, only one gene (LEAFY, Dlo_005438.1) showed significant upregulation during T1 to T2 in 'SX', whereas AP2 (Dlo_000287.1) and CONSTANS-like 14 (Dlo_031781.1) displayed significant upregulation during $\mathrm{T} 1$ to $\mathrm{T} 2$. The remaining genes were significantly and differentially expressed during $\mathrm{T} 2$ to $\mathrm{T} 3$ in 'SX', including phytochrome (phy), embryonic flower 1, SOC1-1, CONSTANS-like, TCP, REVEILLE, GID, FKF1 and cryptochrome (cry). Most of these flowering-related DEGs are associated with photoperiod and the circadian clock pathways, such as the COL gene, APRRs, FKF1, phytochrome, and TF TCPs. Moreover, only one gene (LEAFY, Dlo_005438.1) involved in the floral meristem 


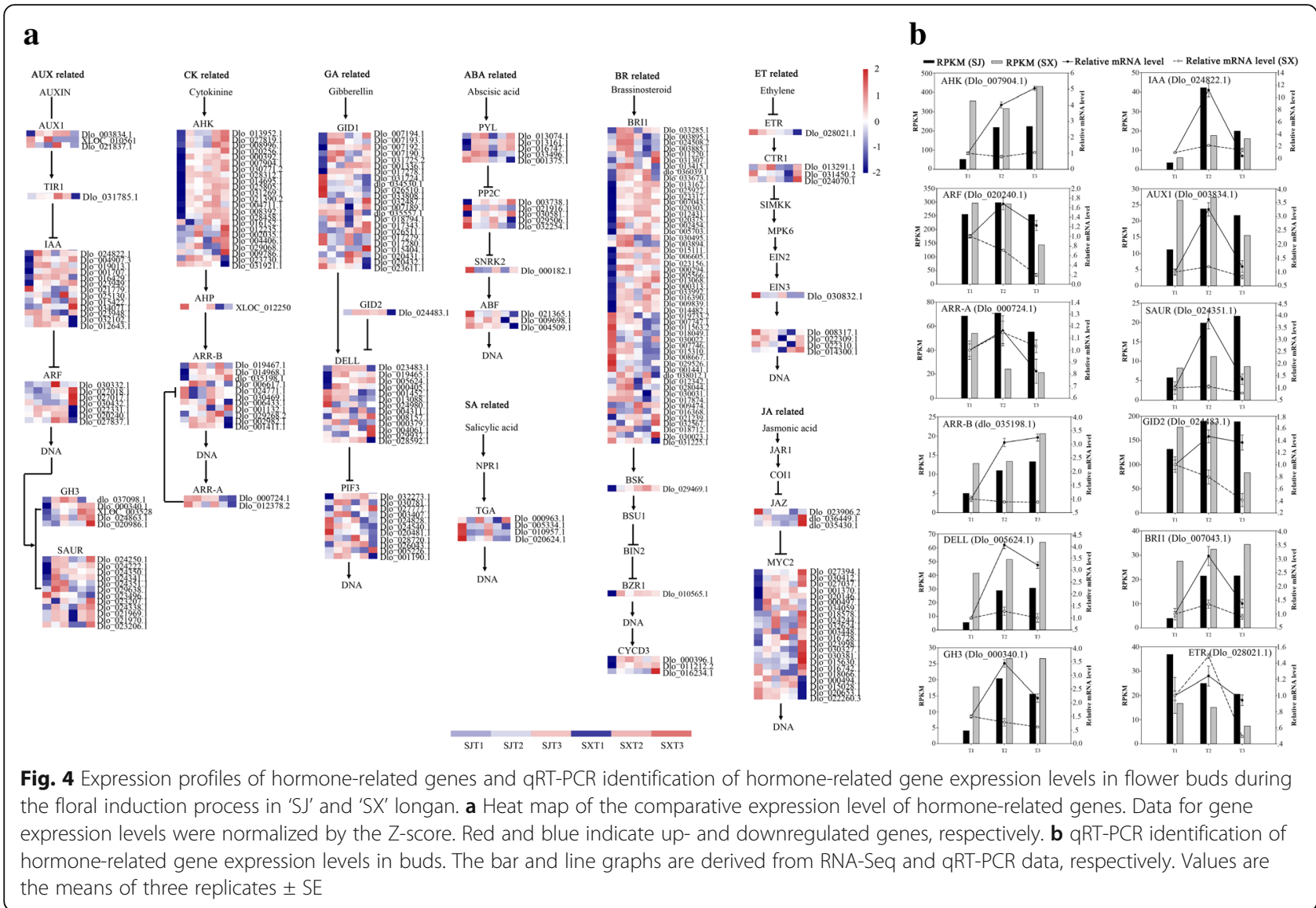

pathway was significantly upregulated during $\mathrm{T} 1$ to $\mathrm{T} 2$ in 'SX', yet it showed no apparent change during the flower induction process in 'SJ'. However, TFL1 and FLC, which controls the PF trait in Arabidopsis and strawberry, did not show differential expression during the floral induction process in our study. These results indicate that photoperiod and circadian clock and floral meristem pathways have significant roles in regulating PF trait formation in longan.

\section{Identification of transcription factor-related DEGs during flower induction}

In the present study, we found many DEGs encode TFs such as GRAS, MADs, NAC and MYB TFs, which are involved in the regulation of floral transition [36-39]. As shown in Fig. 6, 19 GRAS TFs displayed differential expression during flower induction in 'S' and 'SX', including eight genes that were significantly up- or downregulated during T1 to $\mathrm{T} 2$ in 'SJ' and ten with differential expression during flower induction in 'SX'. In addition, one GRAS family gene (Dlo_001452.1) showed significant downregulation in these two cultivars (Additional file 14). Fifteen DEGs are MADs TFs, including one gene (Dlo_021466.1) that displayed significant upregulation during $\mathrm{T} 1$ to $\mathrm{T} 2$ in 'SJ', one gene (Dlo_002044.1) that was significantly downregulated during T2 to T3 of 'SX', eight genes that displayed differential expression during flower induction in 'SX', and two genes (Dlo_031930.1 and Dlo_008014.1) that exhibited a constant increase in both cultivars (Additional file 14). Thirty-one NAC-like family members displayed differential expression during flower induction in 'SJ' and 'SX', including five (dlo_035605.1, Dlo_010076.1, Dlo_032492.2, Dlo_ 003709.1 and Dlo_005892.1) significantly downregulated genes during T1 to T2 in 'SJ', and seven (Dlo_028054.1, Dlo_012365.1, Dlo_022129.1, Dlo_ 012309.3, Dlo_028436.1, Dlo_020074.1 and Dlo_ 005893.1) common genes that were significantly downregulated during $\mathrm{T} 1$ to $\mathrm{T} 2$ in both cultivars; the remaining 19 genes displayed differential expression during flower induction in 'SX' (Additional file 14). Moreover, MYB family genes displayed differential expression during flower induction in 'SJ' and 'SX', including 19 and eight with significant downregulation during T1 to T2 in 'SJ', 17 with differential expression during flower induction in 'SX', and 34 with differential 


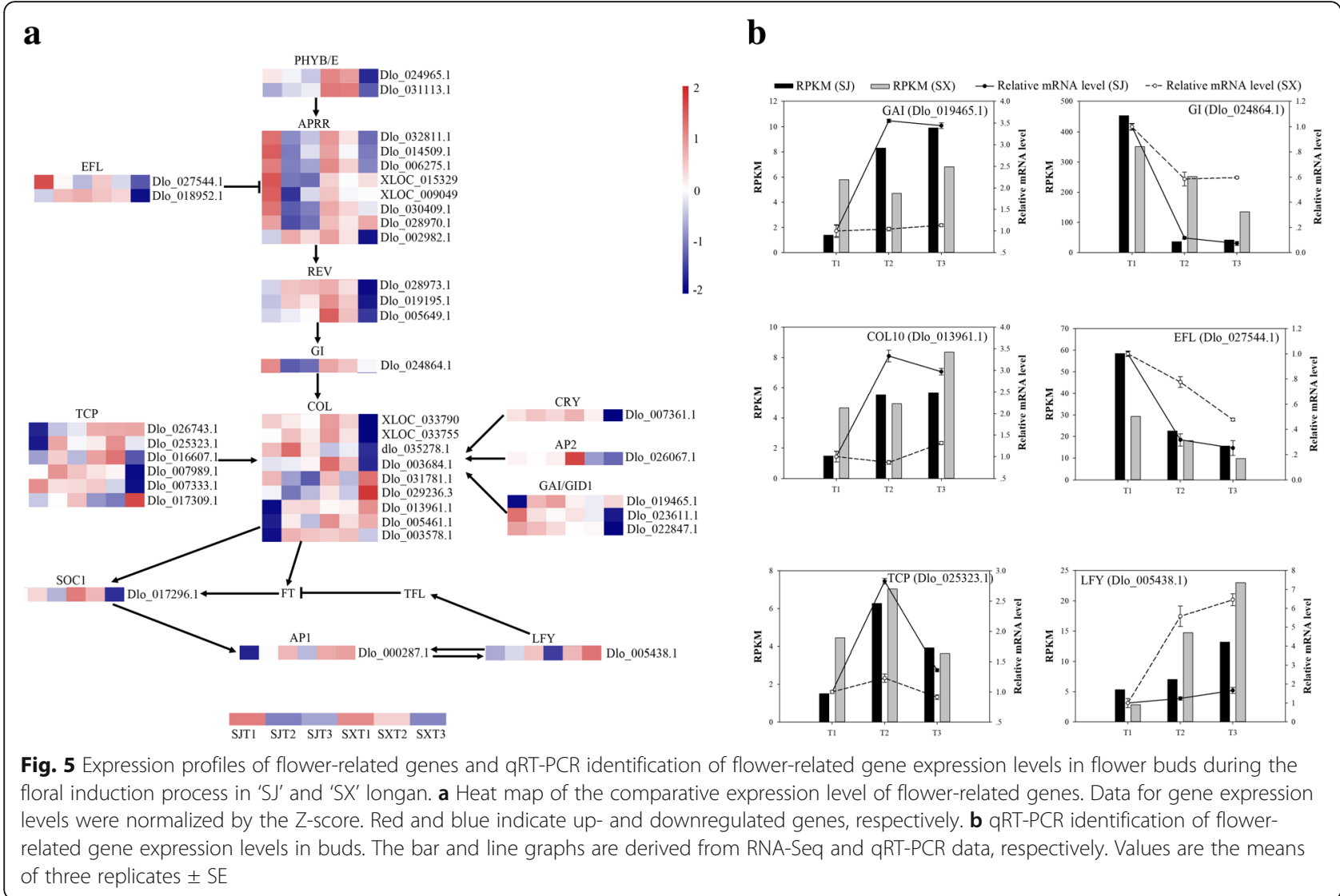

expression during flower induction in both cultivars (Additional file 14). DEGs likely involved in the longan PF trait are summarized in Fig. 7.

\section{Verification of RNA-Seq results by qRT-PCR}

To further validate the RNA-Seq results, 38 DEGs involved in sugar metabolism, hormone signal transduction, photoperiod and circadian clock pathways were selected for parallel qRT-PCR-based expression analysis in 'SJ' and 'SX' (Figs. 3b, 4b, 5b, and 6b). The qRT-PCR data were in agreement with the RNA-Seq data, confirming the accuracy of our transcriptomic analysis (Pearson correlation coefficient $\mathrm{R}^{2}=0.9782$; Additional file 15).

\section{Discussion}

It is important to study transcriptional regulation and its effect on phenotype [40]. Although several studies have examined flowering genes in 'SJ' using RNA-Seq technology [4, 5], the molecular mechanism underlying $\mathrm{PF}$ traits in 'SJ' remains unknown. Thus, to clarify the genetic basis for PF formation in 'SJ', comparative transcriptome analysis was performed in this study. A total of 853.72 M clean reads comprising $125.08 \mathrm{~Gb}$ were obtained. After comparing these data with the longan genome, 27,266 known genes and 1913 new genes were identified [24]. To monitor the transcriptional changes in these two longan cultivars ('SJ' and 'SX') during the floral induction process, DEGs were compared between these cultivars during flower induction. A total of 6150 DEGs were identified during the floral induction process in 'SJ', including 4621 in the T1 to T2 transition and 386 in the T2 to T3 transition; in 'SX' 1880 DEGs in the T1 to $\mathrm{T} 2$ transition and 3488 in the $\mathrm{T} 2$ to $\mathrm{T} 3$ transition were found (Fig. 1). These results show that DEGs related to PF were mainly enriched in the T1 to T2 transition, which is consistent with a previous study suggesting that red buds (T2 stage) are a signal of longan floral initiation [41]. We found that DEGs in the starch and sucrose metabolism pathway were only significantly enriched in profile 6 of 'SJ', which showed increased expression at the $\mathrm{T} 1$ to $\mathrm{T} 2$ transition, and that DEGs associated with plant hormone signal transduction, which were upregulated or downregulated during $\mathrm{T} 1$ to T2, were significantly clustered in profiles 1 and 6 in 'SJ' (Fig. 2). These results suggest that sugar and hormone pathways may play crucial roles in longan PF trait formation. Similar results have been reported for rose species [8].

The transition from dormancy to active bud growth requires sufficient energy [42]. As the main source of energy, carbohydrates are key for several plant developmental stages, including flower induction [43]. For 


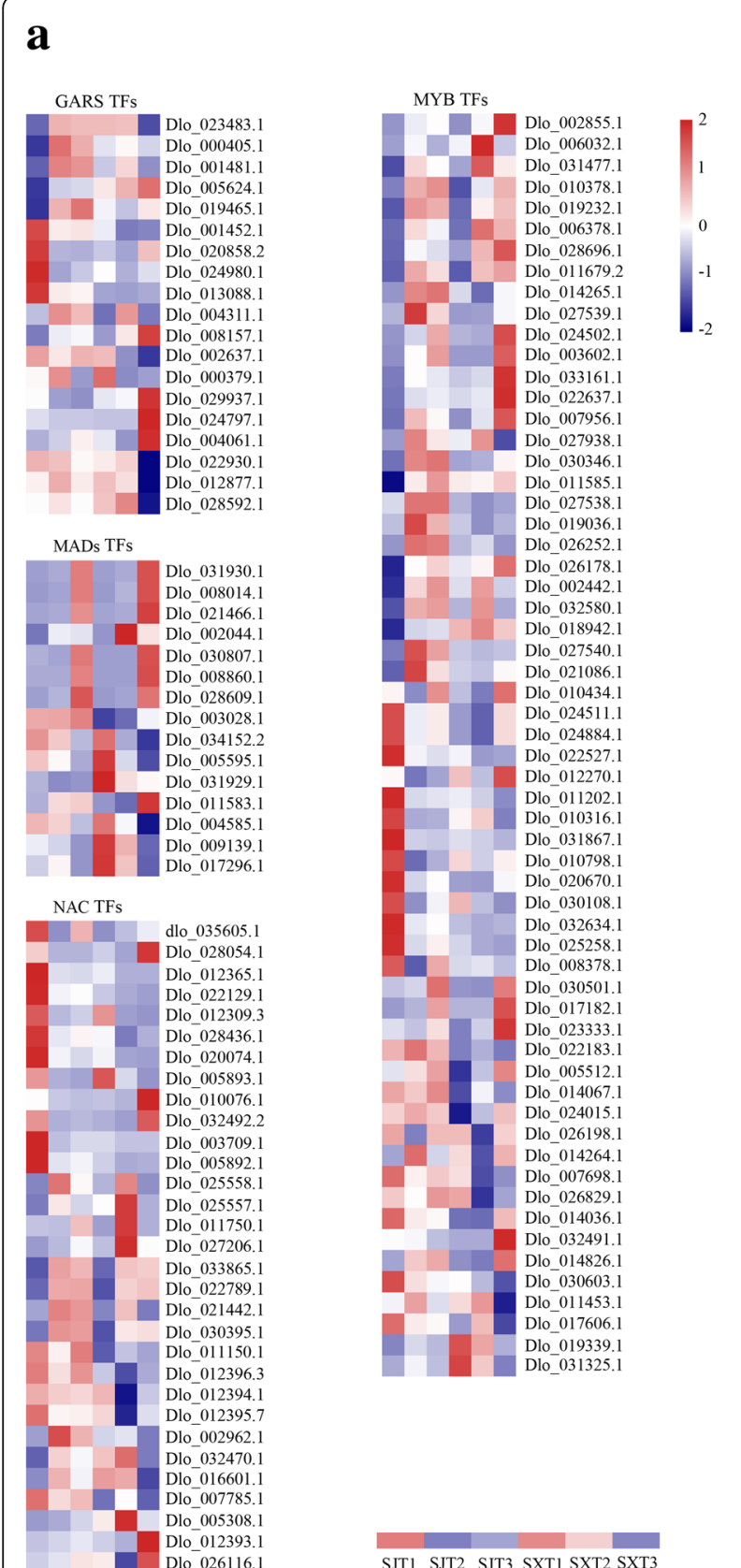

b
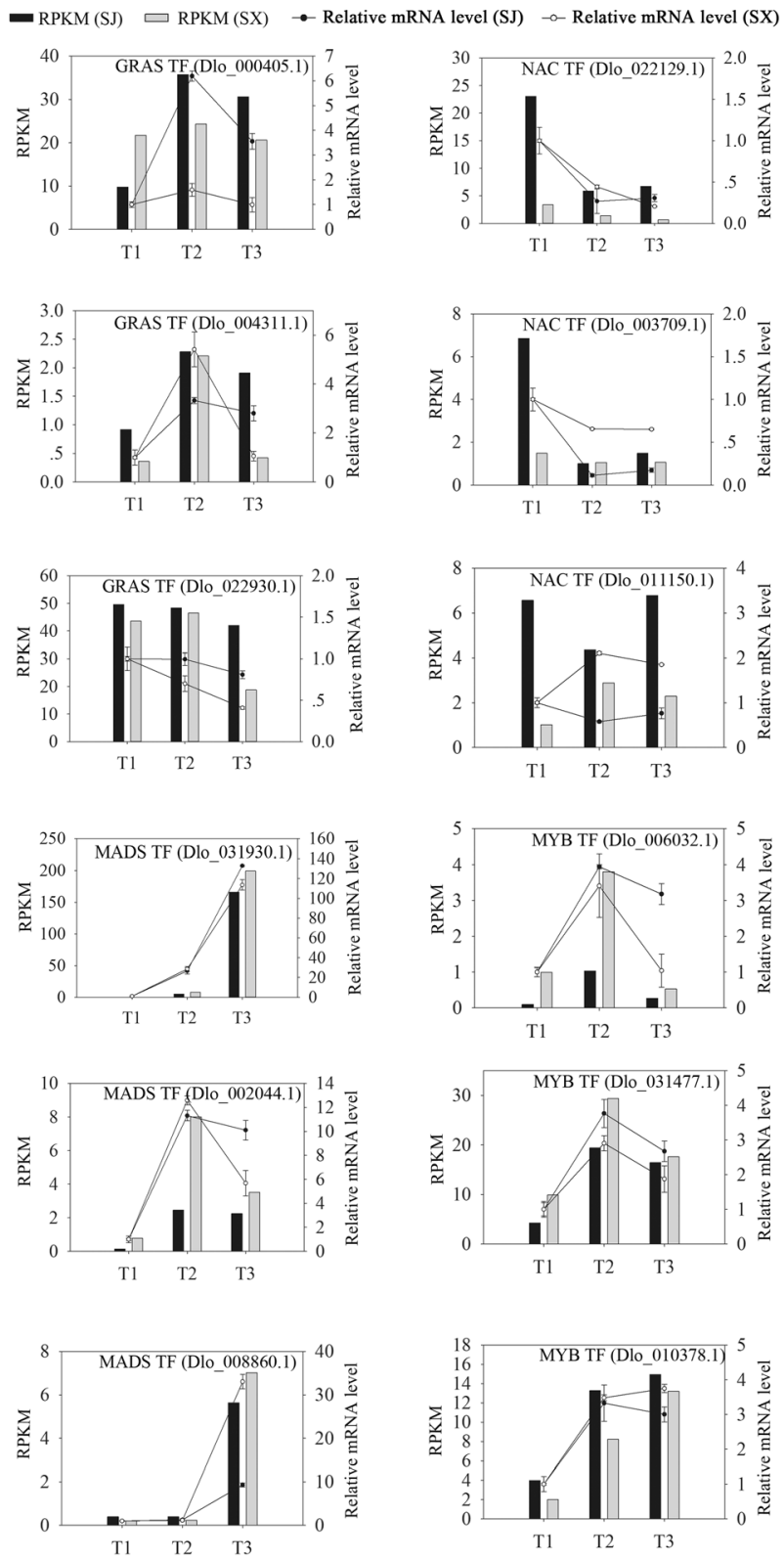

Fig. 6 Expression profiles of transcription factor-related genes and GRT-PCR identification of transcription factor-related gene expression levels in flower buds during the floral induction process in 'SJ' and 'SX' longan. (a) Heat map of the comparative expression level of transcription factorrelated genes. Data for gene expression levels were normalized by the Z-score. Red and blue indicate up- and downregulated genes, respectively. b qRT-PCR identification of transcription factor-related gene expression levels in buds. The bar and line graphs are derived from RNA-Seq and qRT-PCR data, respectively. Values are the means of three replicates \pm SE

example, the rate of leaf sucrose export increases during floral induction in Arabidopsis [44]. Similarly, increases in stored carbohydrates promote floral initiation in several horticultural trees. In fact, girdling, which has been reported to increase levels of stored carbohydrates, promotes flowering intensity in olive [45], lychee [46], and citrus [47]. In contrast, the contents of soluble sugar and starch in the buds of PF rose were higher than those in the buds of SF rose [8]. These results indicate that a suitable concentration of carbohydrates may be associated with floral induction and PF traits in plants. Consistent with such changes in carbohydrate contents, expression of genes related to starch and sucrose metabolism also displayed differential expression during floral induction. For 


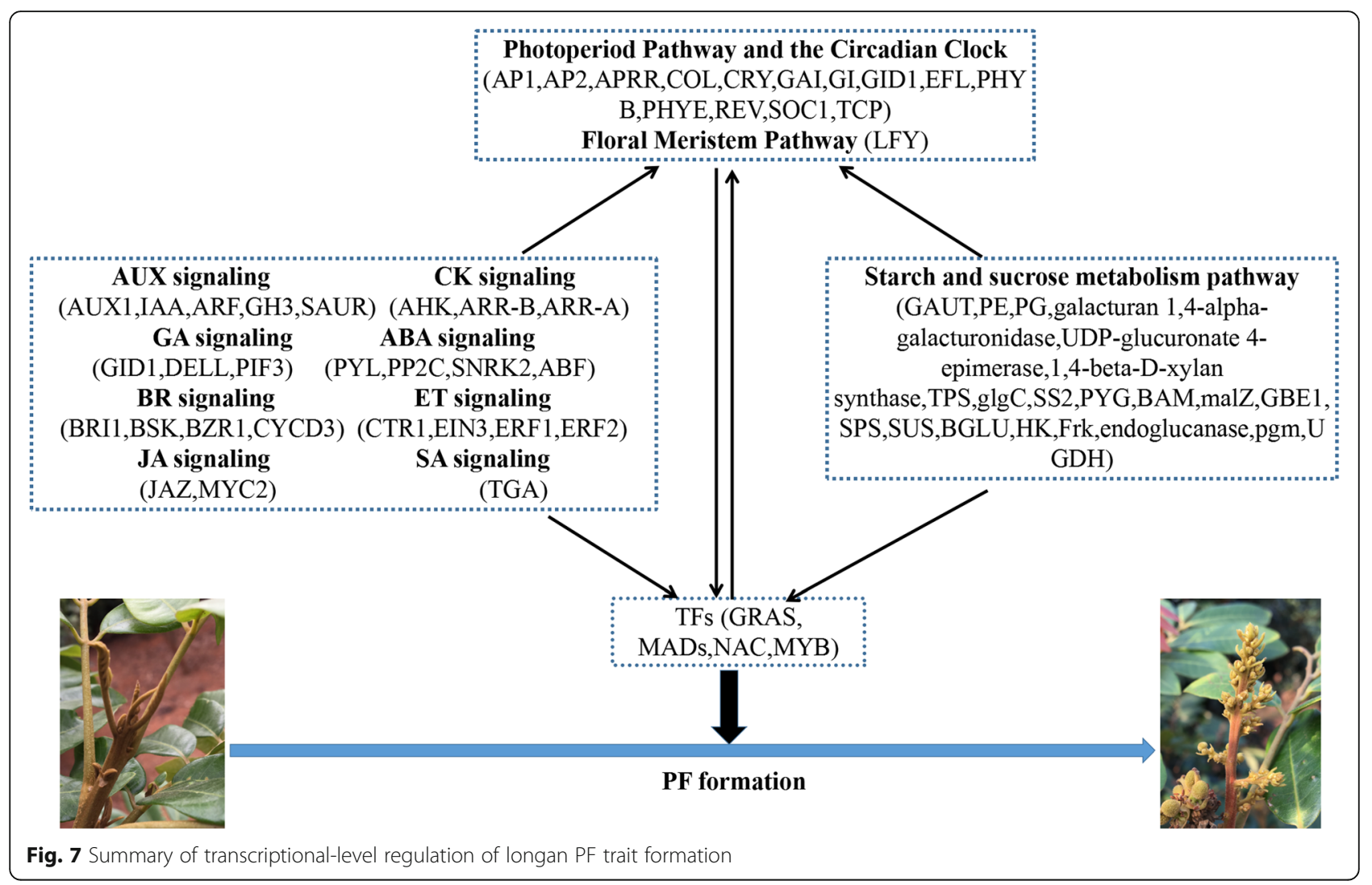

instance, levels of TPS1, fructose-1,6-bisphosphatase I $(F B P)$, and sucrose synthase (SUS) decrease during the floral transition process in SF rose, whereas levels of granule bound starch synthase 1 (GBSS1) and starch synthase 2 (SS2) increase [8]. In apple, TPS displays a gradual increase during flower induction [48]. In addition, expression of sugar biosynthesis-related genes such as SS2, GBSS, and SUS increase during floral induction in Litchi and Doritaenopsis $[49,50]$. Compared to 'SX', almost all DEGs related to starch and sucrose metabolism pathways were significantly altered during the $\mathrm{T} 1$ to $\mathrm{T} 2$ transition in 'SJ', whereas only 5 starch and sucrose-related DEGs changed significantly during the T2 to T3 transition. In contrast, almost all starch and sucrose-related DEGs in 'SX' were significantly altered during the $\mathrm{T} 2$ to $\mathrm{T} 3$ transition. This result suggests that starch and sucrose metabolism pathways might play vital roles in regulating the floral transition in these two longan genotypes, especially with regard to the emergence of floral primordia in PF longan cultivars. For example, starch synthase 2 and pgm, which are involved in starch metabolism, were only downregulated in the SJT1 and SJT2 comparisons. As a proxy for plant carbohydrate status, the signaling molecule trehalose-6-phosphate (T6P) plays a crucial role in the regulation of flowering [51]: TPS1 is necessary for normal vegetative growth and floral induction in Arabidopsis, and loss of TPS1 leads to extremely late flowering in
Arabidopsis, even under other inducing environmental conditions [51]. However, in this study, TPS1 was only significantly downregulated in 'SJ', indicating that TPS1 may function as an inhibitor of PF traits in longan. Similarly, GBSS1, SS2, and pgm were also only downregulated in 'SJ'. Conversely, the expression levels of several genes, such as SUS and BAM4, related to sugar biosynthesis were only significantly upregulated in 'SJ', indicating that these genes act as activators of PF traits in longan.

The flowering time of plants is determined by endogenous genetic components and various environmental factors, including temperature, stress, phytohormones, and day length [52]. Among these factors, hormone signaling plays a crucial role in the complex regulation of the floral transition [53]. A previous study has shown that changes in the contents of gibberellin $\left(\mathrm{GA}_{3}\right)$, indole acetic acid (IAA) and zeatin ribosides (ZRs) differ during the natural floral differentiation process in 'SJ' and that ethephon and $\mathrm{GA}_{3}$ may promote flower formation and fruit setting in 'SJ' longan [54]. However, limited information is available on the effect of hormone signaling pathways on PF traits in plants. Auxin is an important hormone that participates in several aspects of plant growth and development, including floral induction [55]. In Arabidopsis, localized accumulation of auxin, which releases auxin response factor5/monopteros (ARF5/MP) from Aux/IAA repression, is the main reason for the emergence of flowers on 
the flanks of the shoot apical meristem (SAM) [56]. Similar to carbohydrates, the specific concentration of auxin is also a critical factor in floral induction because low auxin levels promote flowering and high concentrations delay flowering [52]. In addition, auxin biosynthesis and signaling pathway-related genes play an important role in flowering. For example, Arabidopsis mutants of ARF2, an auxin-mediated TF, display late flowering [57]. Levels of $G H 3$, which maintains auxin homeostasis and promotes the conversion of auxin to amino acids, may increase during floral induction in SF rose and decrease in PF rose [8]. Similarly, in this study, many genes related to the auxin pathway, such as GH3, LAX (auxin influx carrier), IAA (auxin-responsive protein IAA), and SAUR family genes, were significantly differentially expressed during floral induction (T1 to $\mathrm{T} 2$ transition) in these two longan cultivars that exhibit differing flowering traits (Fig. 3 and Additional file 11). Therefore, these genes may participate in the promotion of PF traits through effects on the emergence of the floral meristem (FM).

The phytohormone cytokinin (CK) is a regulator of many processes in plants, including cell proliferation and differentiation, shoot and root growth, seed germination, and leaf senescence $[58,59]$. Previous studies have reported that $\mathrm{CK}$ is involved in FM formation and that exogenously applied CK can promote flowering in Arabidopsis [60-63]. During the emergence of floral primordia, Arabidopsis histidine-containing phosphotransfer protein6 (AHP6), a negative regulator of CK signaling, is induced by the MP protein. AHP6 then may diffuse to adjacent sites, where it inhibits meristem initiation, thereby enhancing the auxin phyllotactic pattern [64, 65]. Arabidopsis histidine kinase2 (AHK2), AHK3, and AHK4/CRE1 are three membrane-located receptors that perceive the cytokinin signal [66-68], and lines carrying ahk 2 and $a h k 3$ variant alleles display an early flowering phenotype [58]. In the present study, most CK-related genes were significantly and differentially expressed during the floral induction process in both cultivars (Fig. 3 and Additional file 11). For example, 12 and 1 (Dlo_016152.1) AHK genes displayed significant up- or downregulation during the early floral induction stage in 'SJ', respectively, no significant changes were observed in 'SX' indicating these genes may participate in regulating floral induction in 'SJ'.

GA is an important class of plant hormones involved in many aspects of development [69]. In woody species, a decline in GA is beneficial for floral induction $[9,70]$, and GA inhibits the floral transition in apple by repressing CK responses and signaling [71]. In this study, 7 gibberellin receptor GID1 and 4 DELLA genes were upregulated in the early floral induction stage in 'SJ', and 3 GID1 genes were downregulated. In contrast, 5 GID1 and 2 DELLA genes were upregulated in the early floral induction stage in 'SX', and 2 GID1 genes and one DELLA gene were downregulated. These results suggest that the GA signaling pathway may have different functions during floral induction in these two longan cultivars; however, further analyses are required.

Abscisic acid (ABA) also participates in many developmental processes such as seed development, stresses response, and floral transitions [72], and exogenous applications of $\mathrm{ABA}$ alters flowering time in several species [73]. In apple trees, ABA levels gradually increase in floral buds during the floral transition, indicating a positive role of ABA in flowering. Some ABA signaling-related genes such as $M Y C 2$, Pyrabactin resistance 1-like4 (PYL4), and KIN10 (SnRK2.6) also gradually increase in floral buds during flower induction [71], and when exogenously applied to Arabidopsis, ABA functions as a inhibitor of flowering [74]. Similarly, the ABA level decreases during the floral transition in rose genotypes displaying SF or PF traits. Several ABA-related genes such as PYL12 and SnRK2/2.5 are downregulated, though protein phosphatase 2 C25 (PP2C25) is upregulated, during the floral transition [8]. In contrast, $P Y L$ genes were significantly upregulated but $P P 2 C$ downregulated during the floral transition in 'SJ' and 'SX', indicating that the ABA signaling pathway may be involved in regulating different flowering traits in these two longan cultivars.

The steroid hormone brassinosteroid (BR), which is widely distributed in plants, plays a role in plant growth and development as well as responses to various stresses [75-77]. In addition, previous studies have reported that BR is involved in controlling the floral transition, and BR-deficient and -insensitive mutants are often described as late flowering [75, 78]. In Arabidopsis thaliana, brassinosteroid-insensitive 1 (bril) acts as an enhancer of the late flowering autonomous-pathway mutant LUMINIDEPENDENS $(L D)$, and attenuation of BR signaling enhances $F L C$ expression and delays flowering [79]. Similarly, BSK (Dlo_029469.1), BZR1 (Dlo_010565.1), CYCD3 (Dlo_000396.1 and Dlo_011212.2) and most BRI1-like genes were upregulated in the early floral induction stage in 'SJ' (Fig. 4 and Additional file 12), indicating that the BR signaling pathway may promote PF in longan. However, recent research indicates that the BR pathway TF BZR1 can upregulate FLC expression and consequent floral repression by recognizing and binding to a BRRE cis-element in the first intron of the gene [80]. Consistent with this result, levels of several BRI1-like genes decreased in the early floral induction stage in 'ST'.

Other hormone pathways such as the ethylene (ET), salicylic acid (SA), and methyl jasmonate (MeJA) signaling also affect floral induction [52, 81]. For instance, SA-deficient mutants exhibit late flowering under both long-day (LD) and short-day (SD) conditions by inducing expression of $F L C$ [82]. MeJA appears to delay flowering in Arabidopsis and Triticum aestivum, and 
mutation of bHLH TF genes represses JA signaling, resulting in late blooms; the JA receptor mutant coil also shows early flowering [83, 84]. Mutants of Ser/Thr kinases CTR1 (Atctr1), which act as inhibitors of ethylene signaling, exhibit late flowering, whereas mutations in OsERS2, OsETR2, and OsETR3 cause enhanced ethylene sensitivity and early flowering, indicating that ethylene inhibits flowering in Arabidopsis and rice [85, 86]. In the present study, CTR1, jasmonic acid-amido synthetase JAR1, MYC2, and $b H L H T F$ genes were upregulated during early floral induction in 'ST', and ERF-1 (ethylene response factor) and TGA1 TF genes were downregulated during early floral induction in both 'ST' and 'SX'. In summary, hormone biosynthesis and signaling pathways may affect PF trait formation in longan. However, further research is needed on the specific functions of hormone signaling in regulating PF trait formation in longan because of the complex networks of crosstalk between different hormones and reported contradictions between various species.

Flowering is a complex process of morphogenesis that is controlled by a complex network involving vernalization, autonomous, photoperiod, GA-dependent, and aging pathways. These pathways, which are both independent and cross-linked, generate positive and negative feedback and combine flowering signals into several key floral integrators (e.g., FT, TSF and SOC1), activate floral meristem identify genes (e.g., $L F Y$ and $A P 1$ ), and promote flowering [52, 87, 88]. The genetic control of PF has been studied in several model plants such as Arabidopsis, strawberry and rose [16-20], though the complex regulatory mechanisms involved in PF trait formation in fruit trees are still unknown. Although most flower-related genes are conserved among species $[89,90]$, the function of many of these genes differ between model plants and woody plants [15]. For instance, TFL1 and FLC, which control PF in Arabidopsis and strawberry showed no differential expression during the floral induction process in our study, possibly indicating a different mechanism of PF trait formation between longan and model plants. Thus, it is necessary to investigate the regulatory mechanisms involved in PF trait formation in fruit trees, which is directly linked to production potential. In this study, 39 flower-related genes differentially expressed during floral induction between 'SJ' and 'SX' were identified (Fig. 5 and Additional file 13). Most are associated with the photoperiod and circadian clock pathways, such as the $C O L$ gene, APRRs, FKF1, phytochrome, and TF TCPs, indicating that photoperiod and circadian clock-mediated floral induction are essential for determination of the floral fate in 'SJ' longan. Surprisingly, no clear differential expression of genes associated with vernalization and autonomous pathways, which might be important for fruit trees, was found during floral induction between the two longan cultivars. $\mathrm{CO}$ acts as a network hub, integrating various external and internal signals into the photoperiod and circadian clock pathways [91]. As a key gene in the photoperiod pathway, $\mathrm{CO}$ controls the floral transition by directly inducing expression of the $F T$ gene [92]. The transcriptional level of $C O$ is affected by GIGANTEA (GI), which participates in the circadian clock pathway [93, 94]. The GI-CO-FT module is the main photoperiod and circadian clock pathways in Arabidopsis and also exists in other plants such as in Populus deltoides and apple [71, 95]. In this study, three CO-like genes (COL2, Dlo_003578.1; COL7, Dlo_005461.1; COL10, Dlo_013961.1) were upregulated during the floral induction process in 'SJ', whereas GI (Dlo_024864.1) displayed an opposite expression pattern, suggesting that these $C O L$ and GI genes may function as activators or suppressors of floral induction in 'SJ'. Similar to COL genes, two teosinte branched 1/cycloidea/proliferating cell nuclear antigen factor (TCP) genes (TCP4-like, Dlo_026743.1; TCP5-like, Dlo_025323.1), which act as CO activators [96], and the GAI gene were upregulated during floral induction in 'SJ'. Pseudo-response regulator (PRR) proteins have the ability to increase $\mathrm{CO}$ binding to the FT promoter, leading to FT transcriptional enhancement and early flowering [97]. In the present study, 5 PRR genes showed changes similar to those of GI, indicating potential functions in PF trait formation in longan. Early flowering (EFL) proteins are a type of circadian clock component with an important role in flowering. For instance, EFL3 inhibits flowering under noninducing photoperiods by blocking the production of GA and expression of $F T 1$ [98]. Jia et al. (2014) found that the expression level of ELF4 (Unigene4309) increased in 'ST' compared with 'Lidongben', indicating that ELF4 may be involved in PF traits and that ELF4 may be a key gene. Similarly, the EFL4-like gene (Dlo_027544.1) functions as a suppressor of floral induction in 'SJ'. APETALA2 (AP2), a target of miR172 that has been implicated in floral stem cell control [99], was downregulated during floral induction in 'SX', as was COL14, indicating a promoting role in this process. Guo et al. [8] reported similar results. $L F Y$, a plant-specific TF, is the central floral meristem identity gene [100], and the expression level of $L F Y$ is an important determinant of flower initiation [101]. $L F Y$ acts as a key regulator in the integration of flowering signaling pathways, controls the transition from the inflorescence meristem to the floral meristem, and regulates flowering time [62]. During the emergence of floral primordia, $L F Y$ is directly induced by the MP protein, and it then activates expression of the downstream regulator axillary meristems1 (RAX1) and simultaneously inhibits expression of Arabidopsis response regulator7 (ARR7), thereby activating CK signaling and eventually promoting floral meristem formation [102-104]. In the young floral meristem, the MADS-box genes cauliflower $(C A L)$, short vegetative 
phase (SVP), agamous-like 24 (AGL24), and apetala1 (AP1) are induced by $L F Y$ to specify floral identify. Indeed, a feed-forward regulatory loop between $A P 1$ and $L F Y$ stabilizes floral identity and inhibits TFL1 to promote the transition from the inflorescence meristem to the floral meristem [62]. However, a recent study showed that in the absence of AP1/CAL activity, TFL1 expression is suppressed by $A P 1$ and directly promoted by $L F Y$, indicating that $L F Y$ has an inhibitory effect on flower formation [105]. Consistent with this study, LFY (Dlo_005438.1) was significantly upregulated during T1 to T2 in 'SX', though it showed no apparent changes during flower induction in 'SJ', suggesting a potential inhibition role in longan PF trait formation.

In addition to the above genes involved in different pathways, several TFs such as GRAS, MADs, NAC and MYB also play a vital role in regulating downstream floral transition genes [36-39]. Previous research showed that some GRAS family genes may be involved in regulating the floral transition in species such as grape and apple $[39,106]$. In our study, 52 GRAS genes were found in the longan genome, and 19 GRAS TFs displayed differential expression during flower induction in 'SJ' and 'SX' (Fig. 6 and Additional file 14). Interestingly, among these 19 GRAS TFs, two RGL1-like genes (Dlo_024980.1 and Dlo_008157.1) and one GAI (Dlo_019465.1) gene are involved in the GA-mediated signaling pathway. Moreover, DELLA proteins may repress the transcriptional activity of the CO TF by direct interaction, and RGL1 is likely involved in sugar metabolism in SF rose [69]. We also identified 109 MADS-box family genes in the longan genome. MADS-box family genes are also involved in plant floral induction; for example, the AGL6 gene (OsMADS6) determines floral meristem and floral organ identity in rice [36]. Among them, 15 of these genes displayed differential expression during flower induction in 'SJ' and 'SX' (Fig. 6 and Additional file 14). NAC participates in floral induction in several species [37, 107], and in longan, 31 of 107 NAC-like family members were differentially regulated during flower induction in 'S' and 'SX' (Fig. 6 and Additional file 14). Seven genes (Dlo_028054.1, Dlo_012365.1, Dlo_022129.1, Dlo_012309.3, Dlo_028436.1, Dlo_020074.1 and Dlo_005893.1) were common, and expression of the remaining genes was significantly altered during flower induction in 'S' and 'SX'. MYB TFs are also essential for floral organ development and play key roles in pollen development in various species such as cotton and Arabidopsis [38, 108]. MYB levels decrease in PF rose during floral transition [8]. Consistent with these results, 19 and eight genes showed significant up- and downregulation, respectively, during T1 to T2 in 'SJ', and 17 genes displayed differential expression during flower induction in 'SX' (Fig. 6 and Additional file 14). Hence, these TFs may participate in the formation of longan PF traits.

\section{Conclusion}

Research to date on the regulatory mechanisms involved in PF has mainly focused on model plants. As an important trait that is directly linked to production potential, it is necessary to elucidate the regulatory mechanisms involved in PF trait formation in fruit trees. In this study, comparative transcriptome analysis was performed using two longan cultivars that exhibit differing flower phenotypes during the floral induction process. According to our study, the transcriptional differences in 'SJ' are mainly concentrated at the early floral induction stage, which is consistent with a previous study showing that red buds (T2 in our study) are the signal for floral induction. Comparing these two longan genotypes, the transcriptional landscape of the T1 to T2 transition differed greatly with regard to key hormones, circadian rhythm, and sugar pathways, as well as TFs. Almost all of the flowering-related DEGs identified are involved in the photoperiod and circadian clock pathways. Unexpectedly, TFL1 and FLC, which control the PF trait in Arabidopsis and strawberry, showed no significant differential expression during floral induction in our study, possibly indicating a novel mechanism of PF trait formation in 'SJ' longan. In addition, the $L F Y$ gene may inhibit PF in 'SJ' longan, though further analyses are required. This study provides a platform for understanding the molecular mechanisms underlying changes between PF and SF longan genotypes and will aid future studies on PF trait mechanisms of evergreen fruit trees.

\section{Additional files}

Additional file 1: List of primers used in this study. (XLSX $12 \mathrm{~kb}$ )

Additional file 2: The sequencing and mapped results obtained from each sample. (XLS $22 \mathrm{~kb}$ )

Additional file 3: Identification of known genes and new genes in the transcriptome data for eighteen samples. (XLSX $11 \mathrm{~kb}$ )

Additional file 4: DEGs between 'SJ' and ' $S X^{\prime}$ during floral induction. (XLS 11846 kb)

Additional file 5: Significantly enriched GO term analysis of DEGs between 'SJ' and 'SX' during floral induction. (XLSX 22 kb)

Additional file 6: Significantly enriched KEGG pathway analysis of DEGs between 'SJ' and 'SX' during floral induction. (XLSX 15 kb)

Additional file 7: DEGs in 'SJ' during floral induction. (XLS 7620 kb) Additional file 8: DEGs in 'SX' during floral induction. (XLS $7771 \mathrm{~kb}$ ) Additional file 9: GO terms significantly overrepresented in the eight enriched profiles of gene expression versus the reference set in ' $\mathrm{SJ}$ ' and 'SX'. (XLSX 71 kb)

Additional file 10: KEGG pathways significantly overrepresented in the eight enriched profiles of gene expression versus the reference set in 'SJ' and 'SX'. (XLSX $20 \mathrm{~kb}$ )

Additional file 11: Sugar-related DEGs in two longan cultivars during the floral induction process. Red indicates the genes that showed upregulated expression; blue indicates the genes that showed downregulated expression. (XLSX $48 \mathrm{~kb}$ ) 
Additional file 12: Hormone-related DEGs in two longan cultivars during the floral induction process. Red indicates genes that showed upregulated expression; blue indicates genes that showed downregulated expression. (XLSX $107 \mathrm{~kb})$

Additional file 13: Flower-related DEGs in two longan cultivars during the floral induction process. Red indicates genes that showed upregulated expression; blue indicates genes that showed downregulated expression. (XLSX $26 \mathrm{~kb}$ )

Additional file 14: GARS, NAC, MADs, MYB TFs in two longan cultivars during the floral induction process. Red indicates genes that showed upregulated expression; blue indicates genes that showed downregulated expression. (XLSX $125 \mathrm{~kb}$ )

Additional file 15: Linear relationships between GRT-PCR data and RNASeq data of related genes. The $x$-axis indicates the qRT-PCR $\log _{2}$ expression ratios; the $y$-axis indicates the RNA-Seq data ratios. (TIF $262 \mathrm{~kb}$ )

\section{Abbreviations}

DEG: Differentially expressed gene; FDR: False discovery rate; GO: Gene Ontology; KEGG: Kyoto Encyclopedia of Genes and Genomes; NCBI: National Center of Biotechnology Information; qRT-PCR: quantitative real-time reverse transcription polymerase chain reaction; RNA-Seq: RNA sequencing; RPKM: Reads per kilobase of exon model per million mapped reads

\section{Acknowledgements}

We would like to thank the reviewers for their helpful comments on the original manuscript. Our gratitude also goes to Pro. Lin and Lai who shared the longan genome and GENE DENOVO Company for their assistance in analyzing the transcriptome data.

\section{Funding}

This work was supported by the Natural Science Foundation of China (31572087), the China Litchi and Longan Industry Technology Research System (CARS-32-02), the Guangdong Natural Science Foundation (2018A0303070021), the Central Public-interest Scientific Institution Basal Research Fund for Chinese Academy of Tropical Agricultural Sciences (No. 1630062019014), the Science and Technology Program of Guangzhou (201604020188), and the Natural Science Foundation of Hainan Province (317243 and 317241). The funders had no role in the design of the study or the collection, analysis or interpretation of the data or the writing of the manuscript.

\section{Availability of data and materials}

The longan genome sequences were downloaded from NCBI Sequence Read Archive (SRA315202). RNA-Seq data for organ expression profiles were downloaded from NCBI Sequence Read Archive (GSE84467).

\section{Authors' contributions}

DJ and SS conceived and designed the experiments and drafted the manuscript. DJ and XS performed the experiments. BS and LL analyzed the data. YW prepared the CDNA samples for sequencing. CL, JX and YW provided helpful comments on the manuscript. All authors read and approved the final version of the manuscript.

\section{Ethics approval and consent to participate}

Not applicable.

\section{Consent for publication}

Not applicable.

\section{Competing interests}

The authors declare that they have no competing interests.

\section{Publisher's Note}

Springer Nature remains neutral with regard to jurisdictional claims in published maps and institutional affiliations.

\section{Author details}

${ }^{1}$ Key Laboratory of Tropical Fruit Biology (Ministry of Agriculture), South Subtropical Crops Research Institute, Chinese Academy of Tropical
Agricultural Sciences, Zhanjiang 524091, China. ${ }^{2}$ College of Horticulture, South China Agricultural University, Guangzhou 510642, China. ${ }^{3}$ School of Advanced Agriculture and Bioengineering, Yangtze Normal University, Chongqing 408100, China.

Received: 19 June 2018 Accepted: 17 January 2019

Published online: 11 February 2019

\section{References}

1. Matsumoto TK. Genes uniquely expressed in vegetative and potassium chlorate induced floral buds of Dimocarpus longan. Plant Sci. 2006;170(3): 500-10

2. Wang B, Tan HW, Fang W, Meinhardt LW, Mischke S, Matsumoto T, Zhang D. Developing single nucleotide polymorphism (SNP) markers from transcriptome sequences for identification of longan (Dimocarpus longan) germplasm. Hortic Res. 2015;2(1):14065.

3. Wu YL, Yi GJ, Zhou BR, Zeng J, Huang YH. The advancement of research on litchi and longan germplasm resources in China. Sci Hortic. 2007;114(3):14350 .

4. Jia T, Wei D, Meng S, Allan AC, Zeng L. Identification of regulatory genes implicated in continuous flowering of longan (Dimocarpus longan L.). PLoS One. 2014:9(12):e114568

5. Zhang HN, Shi SY, Li WC, Shu B, Liu LQ, Xie JH, Wei YZ. Transcriptome analysis of 'Sijihua' longan (Dimocarpus longan L.) based on next-generation sequencing technology. J Hortic Sci Biotechnol. 2016;91(2):180-8.

6. Shabala S, Bose J, Hedrich R. Salt bladders: do they matter? Trends Plant Sci. 2014;19(11):687.

7. Turnbull C. Long-distance regulation of flowering time. J Exp Bot. 2011; 62(13):4399-413.

8. Guo X, Yu C, Luo L, Wan H, Li Y, Wang J, Cheng T, Pan H, Zhang Q. Comparative transcriptome analysis of the floral transition in Rosa chinensis 'Old Blush' and R. odorata var. gigantea. Sci Rep. 2017;7(1):6068.

9. Wilkie JD, Sedgley M, Olesen T. Regulation of floral initiation in horticultural trees. J Exp Bot. 2008;59(12):3215-28.

10. Weigel $\mathrm{D}$, Nilsson $\mathrm{O}$. A developmental switch sufficient for flower initiation in diverse plants. Nature. 1995;377(6549):495-500.

11. Böhlenius H, Huang T, Charbonnelcampaa L, Brunner AM, Jansson S, Strauss $\mathrm{SH}$, Nilsson O. CO/FT regulatory module controls timing of flowering and seasonal growth cessation in trees. Berlin Heidelberg: springer; 2006.

12. Hsu CY, Liu Y, Luthe DS, Yuceer C. Poplar FT2 shortens the juvenile phase and promotes seasonal flowering. Plant Cell. 2006;18(8):1846-61.

13. Mao Y, Sun J, Cao P, Zhang R, Fu Q, Chen S, Chen F, Jiang J. Functional analysis of alternative splicing of the FLOWERING LOCUS T orthologous gene in Chrysanthemum morifolium. Hortic Res. 2016:3:16058.

14. Winterhagen $P$, Tiyayon $P$, Samach $A$, Hegele M, Wünsche JN. Isolation and characterization of FLOWERING LOCUS T subforms and APETALA1 of the subtropical fruit tree Dimocarpus longan. Plant Physiol Biochem. 2013;71(2): 184-90.

15. Strauss SH, Brunner AM, Busov VB, Ma C, Meilan R. Ten lessons from 15 years of transgenic Populus research. Forestry. 2004:77(5):455-65.

16. Iwata H, Gaston A, Remay A, Thouroude T, Jeauffre J, Kawamura K, Oyant LH, Araki T, Denoyes B, Foucher F. The TFL1 homologue KSN is a regulator of continuous flowering in rose and strawberry. Plant J. 2012;69(1):116-25.

17. Wang R, Farrona S, Vincent C, Joecker A, Schoof H, Turck F, Alonso-Blanco C, Coupland G, Albani MC. PEP1 regulates perennial flowering in Arabis alpina. Nature. 2009;459(7245):423-7

18. Koskela EA, Mouhu K, Albani MC, Kurokura T, Rantanen M, Sargent DJ Battey NH, Coupland G, Elomaa P, Hytönen T. Mutation in TERMINAL FLOWER1 reverses the photoperiodic requirement for flowering in the wild strawberry Fragaria vesca. Plant Physiol. 2012;159(3):1043.

19. Gaston A, Perrotte J, Lerceteauköhler E, Rousseaugueutin M, Petit A Hernould M, Rothan C, Denoyes B. PFRU, a single dominant locus regulates the balance between sexual and asexual plant reproduction in cultivated strawberry. J Exp Bot. 2013;64(7):1837-48.

20. Perrotte J, Gaston A, Potier A, Petit A, Rothan C, Denoyes B. Narrowing down the single homoeologous FaPFRU locus controlling flowering in cultivated octoploid strawberry using a selective mapping strategy. Plant Biotechnol J. 2016:14(11):2176-89.

21. Chen H, He XH, Zhu JH, Luo C, Jiang JC. Genetic relationships analysis of 37 longan germplasm resources by using ISSR markers. Genomics Appl Biol. 2010;29(2):288-92 
22. Peng J, Xie LJ, Xu BQ, Dang JZ, Li YH, Lu ZH, Zhang SA, Yu ZY, Bai XQ, Cai ZF. Study on biological characters of 'Sijihua' Iongan. Acta Hortic. 2010;863: 249-58.

23. Zhu JH, Pan LM, Qin SQ, Peng HX, Wang Y, Han ZH. Analysis on genetic relations in different ecotypes of longan (Dimocarpus longan) germplasm resources by ISSR markers. J Plant Genet Resour. 2013;14(1):66-70(65).

24. Lin Y, Min J, Lai R, Wu Z, Chen Y, Yu L, Cheng C, Jin Y, Tian Q, Liu Q. Genome-wide sequencing of longan (Dimocarpus longanLour.) provides insights into molecular basis of its polyphenol-rich characteristics. Gigascience. 2017;6(5):1-14

25. Li R, Yu C, Li Y, Lam TW, Yiu SM, Kristiansen K, Wang J, Wang J. SOAP2: an improved ultrafast tool for short read alignment. Bioinformatics. 2009;25(15): 1966-7.

26. Li B, Dewey CN. RSEM: accurate transcript quantification from RNA-Seq data with or without a reference genome. Bmc Bioinformatics. 2011;12(1):323.

27. Anders S, Huber W. Differential expression analysis for sequence count data. Genome Biol. 2010;11(10):R106.

28. Rajkumar AP, Qvist P, Lazarus R, Lescai F, Jia J, Nyegaard M, Mors O, Børglum AD, Li Q, Christensen JH. Experimental validation of methods for differential gene expression analysis and sample pooling in RNA-seq. BMC Genomics. 2015;16(1):548.

29. Ernst J, Bar-Joseph Z. STEM: a tool for the analysis of short time series gene expression data. Bmc Bioinformatics. 2006;7(1):191.

30. Conesa A, Götz S, Garcíagómez JM, Terol J, Talón M, Robles M. Blast2GO: a universal tool for annotation, visualization and analysis in functional genomics research. Bioinformatics. 2005;21(18):3674.

31. Ye J, Fang L, Zheng H, Zhang Y, Chen J, Zhang Z, Wang J, Li S, Li R, Bolund L. WEGO: a web tool for plotting GO annotations. Nucleic Acids Res. 2006; 34(Web Server issue):W293.

32. Kanehisa M, Araki M, Goto S, Hattori M, Hirakawa M, Itoh M, Katayama T, Kawashima S, Okuda S, Tokimatsu T. KEGG for linking genomes to life and the environment. Nucleic Acids Res. 2008;36(Database issue):D480-4.

33. Thimm O, Blasing $O$, Gibon $Y$, Nagel A, Meyer S, Kruger P, Selbig J, Muller LA, Rhee SY, Stitt M. MAPMAN: a user-driven tool to display genomics data sets onto diagrams of metabolic pathways and other biological processes. Plant J. 2010;37(6):914-39.

34. Saeed Al, Sharov V, ., White J, ., Li J, ., Liang W, ., Bhagabati N, ., Braisted J, ., Klapa M, ., Currier T, ., Thiagarajan M, . TM4: a free, open-source system for microarray data management and analysis. Biotechniques 2003, 34(2):374-378.

35. Jue D, Sang X, Liu L, Shu B, Wang Y, Xie J, Liu C, Shi S. The UbiquitinConjugating Enzyme Gene Family in Longan (Dimocarpus longan Lour.): Genome-Wide Identification and Gene Expression during Flower Induction and Abiotic Stress Responses. Molecules. 2018;23(3):662.

36. Zhang D. The AGL6-like gene OsMADS6 regulates floral organ and meristem identities in rice. Cell Res. 2010;20(3):299.

37. Yoo SY, Kim Y, Kim SY, Lee JS, Ji HA. Control of flowering time and cold response by a NAC-domain protein in Arabidopsis. PLoS One. 2007;2(7):e642.

38. Xu Y, lacuone S, Li SF, Parish RW. MYB80 homologues in Arabidopsis, cotton and Brassica: regulation and functional conservation in tapetal and pollen development. BMC Plant Biol. 2014;14(1):278.

39. Fan S, Zhang D, Gao C, Zhao M, Wu H, Li Y, Shen Y, Han M. Identification, classification, and expression analysis of GRAS gene family in Malus domestica. Front Physiol. 2017;8:253.

40. Lin Y, Lai Z. Comparative Analysis Reveals Dynamic Changes in miRNAs and Their Targets and Expression during Somatic Embryogenesis in Longan (Dimocarpus longan Lour.). PLoS One. 2013;8(4):e60337.

41. Qiu J, Dingyao WU, Zhang H: A Study on Flower Differentiation of 'Shixia'Longan (Dimocarpus longana Lour. cv. Shixia). Journal of South China Agricultural University. 2001;22(1):27-30.

42. Zhuang W, Gao Z, Wang L, Zhong W, Ni Z, Zhang Z. Comparative proteomic and transcriptomic approaches to address the active role of GA4 in Japanese apricot flower bud dormancy release. J Exp Bot. 2013;64(16): 4953-66.

43. Gibson SI. Control of plant development and gene expression by sugar signaling. Curr Opin Plant Biol. 2005;8(1):93-102.

44. Corbesier L, Lejeune P, Bernier G. role of carbohydrates in the induction of flowering in Arabidopsis thaliana: comparison between the wild type and a starchless mutant. Planta. 1998;206(1):131-7.

45. Lavee S, Haskal A, Tal YB. Girdling olive trees, a partial solution to biennial bearing. I. Methods, timing and direct tree response. Journal of Pomology \& Horticultural Science. 1983;58(2):209-18.
46. Menzel CM, Simpson DR. Lychee nutrition: a review. Sci Hortic. 1987;31(3): 195-224.

47. Goldschmidt EE, Aschkenazi N, Herzano Y, Schaffer AA, Monselise SP. A role for carbohydrate levels in the control of flowering in citrus. Sci Hortic. 1985; 26(2):159-66.

48. Lastdrager J, Hanson J, Smeekens S. Sugar signals and the control of plant growth and development. J Exp Bot. 2014;65(3):799.

49. Qin Q, Kaas Q, Zhang C, Zhou L, Luo X, Zhou M, Sun X, Zhang L, Paek KY, Cui $Y$. The cold awakening of Doritaenopsis 'tinny tender' orchid flowers: the role of leaves in cold-induced bud dormancy release. J Plant Growth Regul. 2012;31(2):139-55.

50. Zhang $H$, Shen J, Wei $Y$, Chen $H$. Transcriptome profiling of litchi leaves in response to low temperature reveals candidate regulatory genes and key metabolic events during floral induction. BMC Genomics. 2017;18(1):363.

51. Wahl V, Ponnu J, Schlereth A, Arrivault S, Langenecker T, Franke A, Feil R, Lunn JE, Stitt M, Schmid M. Regulation of flowering by Trehalose-6phosphate signaling in Arabidopsis thaliana. Science. 2013;339(6120):704-7.

52. Cho LH, Yoon J, An G. The control of flowering time by environmental factors. Plant J. 2017:90(4):708-19.

53. Davis SJ. Integrating hormones into the floral-transition pathway of Arabidopsis thaliana. Plant Cell Environ. 2009;32(9):1201-10.

54. Peng J, Cai ZF, Deng HG, Zeng SY, Xi JB, Huang WD, Gu M, Xu BQ, Dang JZ, $\mathrm{Li}$ YH. Study on the flowering and fruiting Phenological law of 'Sijihua' Longan (Dimocarpus longan) and its regulation. In: the third international conference symposium on longan, lychee and other fruit trees in sapindaceae family, vol. 2008; 2008. p. 259-66

55. Yamaguchi N, Wu MF, Winter CM, Berns MC, Nole-Wilson S, Yamaguchi A, Coupland G, Krizek BA, Wagner D. A molecular framework for auxinmediated initiation of flower primordia. Dev Cell. 2013;24(3):271.

56. Pierrejerome $\mathrm{E}$, Moss BL, Nemhauser JL. Tuning the auxin transcriptional response. J Exp Bot. 2013;64(9):2557-63

57. Okushima Y, Mitina I, Quach HL, Theologis A. AUXIN RESPONSE FACTOR 2 (ARF2): a pleiotropic developmental regulator. Plant J. 2005;43(1):29-46.

58. Bartrina I, Jensen $H$, Novak O, Strnad M, Werner T, Schmülling T. Gainof-function mutants of the cytokinin receptors AHK2 and AHK3 regulate plant organ size, flowering time and plant longevity. Plant Physiol. 2017;173(3):1783.

59. Kieber JJ, Schaller GE. Cytokinin signaling in plant development. Development. 2018;145(4):dev149344.

60. Besnard-Wibaut C. Effectiveness of gibberellins and 6-benzyladenine on flowering of Arabidopsis thaliana. Physiol Plant. 1981;53(3):205-12.

61. D'Aloia M, Bonhomme D, Bouché F, Tamseddak K, Ormenese S, Torti S, Coupland G, Périlleux C. Cytokinin promotes flowering of Arabidopsis via transcriptional activation of the FT paralogue TSF. Plant J. 2011;65(6):972-9.

62. Denay G, Chahtane H, Tichtinsky G, Parcy F. A flower is born: an update on Arabidopsis floral meristem formation. Curr Opin Plant Biol. 2017;35:15-22.

63. Dennis ES, Finnegan EJ, Bilodeau P, Chaudhury A, Genger R, Helliwell CA, Sheldon CC, Bagnall DJ, Peacock WJ. Vernalization and the initiation of flowering. Semin Cell Dev Biol. 1996;7(3):441-8.

64. Besnard F, Refahi Y, Morin V, Marteaux B, Brunoud G, Chambrier P, Rozier F, Mirabet V, Legrand J, Lainé S. Cytokinin signalling inhibitory fields provide robustness to phyllotaxis. Nature. 2013;505(7483):417-21.

65. Besnard F, Rozier F, Vernoux T. The AHP6 cytokinin signaling inhibitor mediates an auxin-cytokinin crosstalk that regulates the timing of organ initiation at the shoot apical meristem. Plant Signal Behav. 2014;9(6):e28788.

66. Inoue T, Higuchi M, Hashimoto Y, Seki M, Kobayashi M, Kato T, Tabata S, Shinozaki K, Kakimoto T. Identification of CRE1 as a cytokinin receptor from Arabidopsis. Nature. 2001;409(6823):1060-3.

67. Ueguchi C, Sato S, Kato T, Tabata S. The AHK4 gene involved in the cytokinin-signaling pathway as a direct receptor molecule in Arabidopsis thaliana. Plant Cell Physiol. 2001;42(7):751-5.

68. Yamada H, Suzuki T, Terada K, Takei K, Ishikawa K, Miwa K, Yamashino T, Mizuno T. The Arabidopsis AHK4 histidine kinase is a cytokinin-binding receptor that transduces cytokinin signals across the membrane. Plant Cell Physiol. 2001:42(9):1017-23.

69. Wang H, Pan J, Li Y, Lou D, Hu Y, Yu D. The DELLA-CONSTANS transcription factor cascade integrates gibberellic acid and photoperiod signaling to regulate flowering. Plant Physiol. 2016;172(1):479-88.

70. Koshita Y, Takahara T, Ogata T, Goto A. Involvement of endogenous plant hormones (IAA, ABA, GAs) in leaves and flower bud formation of Satsuma mandarin ( Citrus unshiu Marc.). Sci Hortic. 1999;79(3-4):185-94. 
71. Xing LB, Zhang D, Li YM, Shen YW, Zhao CP, Ma JJ, An N, Han MY. Transcription Profiles Reveal Sugar and Hormone Signaling Pathways Mediating Flower Induction in Apple ( Malus domestica Borkh.). Plant Cell Physiol. 2015;56(10):2052

72. Tsai YL, Gazzarrini S. Trehalose-6-phosphate and SnRK1 kinases in plant development and signaling: the emerging picture. Front Plant Sci. 2014;5(5):119.

73. Zhang DP. Abscisic acid: metabolism, Transport and Signaling. Netherlands: Springer; 2014

74. Wang Y, Li L, Ye T, Lu Y, Chen X, Wu Y. The inhibitory effect of ABA on floral transition is mediated by ABI5 in Arabidopsis. J Exp Bot. 2013;64(2):675-84.

75. Zhao B, Li J. Regulation of brassinosteroid biosynthesis and inactivation. Aust J Bot. 2012:54(10):746-59.

76. Wang ZY, Bai MY, Oh E, Zhu JY. Brassinosteroid signaling network and regulation of Photomorphogenesis. Annu Rev Genet. 2012;46(1):701.

77. Nolan T, Chen J, Yin Y. Cross-talk of Brassinosteroid signaling in controlling growth and stress responses. Biochem J. 2017;474(16):2641-61.

78. Clouse SD. The molecular intersection of brassinosteroid-regulated growth and flowering in Arabidopsis. Proc Natl Acad Sci U S A. 2008;105(21):7345-6.

79. Domagalska MA, Schomburg FM, Amasino RM, Vierstra RD, Nagy F, Davis SJ. Attenuation of brassinosteroid signaling enhances FLC expression and delays flowering. Development. 2007;134(15):2841-50.

80. Li Z, Ou Y, Zhang Z, et al. Brassinosteroid Signaling Recruits Histone 3 Lysine-27 Demethylation Activity to Flowering Locus C Chromatin to Inhibit the Floral Transition in Arabidopsis[J]. Mol Plant. 2018;11(9):1135-46.

81. Iabal N, Khan NA, Ferrante A, Trivellini A, Francini A, MIR K. Ethylene Role in Plant Growth, Development and Senescence: Interaction with Other Phytohormones. Front Plant Sci. 2017:8(9):475.

82. Martínez C, Pons E, Prats G, León J. Salicylic acid regulates flowering time and links defence responses and reproductive development. Plant J. 2004; 37(2):209-17.

83. Diallo AO, Agharbaoui Z, Badawi MA, Alibenali MA, Moheb A, Houde M, Sarhan F. Transcriptome analysis of an mvp mutant reveals important changes in global gene expression and a role for methyl jasmonate in vernalization and flowering in wheat. J Exp Bot. 2014;65(9):2271.

84. Song S, Qi T, Fan M, Zhang X, Gao H, Huang H, Wu D, Guo H, Xie D. The bHLH subgroup IIId factors negatively regulate Jasmonate-mediated plant defense and development. PLoS Genet. 2013;9(7):e1003653.

85. Achard P, Baghour M, Chapple A, Hedden P, Van DSD, Genschik P, Moritz T, Harberd NP. The plant stress hormone ethylene controls floral transition via DELLA-dependent regulation of floral meristem-identity genes. Proc Natl Acad Sci U S A. 2007;104(15):6484-9.

86. Qin W, Wei Z, Yin Z, Wen CK. Rice CONSTITUTIVE TRIPLE-RESPONSE2 is involved in the ethylene-receptor signalling and regulation of various aspects of rice growth and development. J Exp Bot. 2013;64(16):4863-75.

87. Bouché F, Lobet G, Tocquin P, Périlleux C. FLOR-ID: an interactive database of flowering-time gene networks in Arabidopsis thaliana. Nucleic Acids Res. 2015;44(D1):D1167-71.

88. Srikanth A, Schmid M. Regulation of flowering time: all roads lead to Rome. Cell Mol Life Sci. 2011;68(12):2013-37.

89. Albani M C, Coupland G. Comparative analysis of flowering in annual and perennial plants.[J]. Current Topics in Developmental Biology, 2010;91(10): 323-48.

90. Blümel $M$, Dally $N$, Jung C. Flowering time regulation in crops — what did we learn from Arabidopsis? Curr Opin Biotechnol. 2015;32:121-9.

91. Shim JS, Kubota A, Imaizumi T. Circadian clock and photoperiodic flowering in Arabidopsis: CONSTANS is a hub for signal integration. Plant Physiol. 2016;173(1):01327.02016.

92. Valverde F, Mouradov A, Soppe W, Ravenscroft D, Samach A, Coupland G. Photoreceptor regulation of CONSTANS protein in photoperiodic flowering. Science. 2004;303(5660):1003-6.

93. Fowler S, Lee K, Onouchi H, Samach A, Richardson K, Morris B, Coupland G, Putterill J. GIGANTEA: a circadian clock-controlled gene that regulates photoperiodic flowering in Arabidopsis and encodes a protein with several possible membrane-spanning domains. EMBO J. 1999;18(17):4679-88.

94. Park DH, Somers DE, Kim YS, Choy YH, Lim HK, Soh MS, Kim HJ, Kay SA, Nam HG. Control of circadian rhythms and photoperiodic flowering by the Arabidopsis GIGANTEA gene. Science. 1999;285(5433):1579-82.

95. Böhlenius H, Huang T, Charbonnelcampaa L, Brunner AM, Jansson S, Strauss $\mathrm{SH}$, Nilsson O. CO/FT regulatory module controls timing of flowering and seasonal growth cessation in trees. Science. 2006;312(5776):1040-3.
96. Kubota A, Ito S, Shim JS, Johnson RS, Song YH, Breton G, Goralogia GS, Kwon MS, Laboy CD, Koyama T. TCP4-dependent induction of CONSTANS transcription requires GIGANTEA in photoperiodic flowering in Arabidopsis. PLoS Genet. 2017:13(6):e100E6856.

97. Hayama R, Sarid-Krebs L, Richter R, Fernández V, Jang S, Coupland G. PSEUDO RESPONSE REGULATORs stabilize CONSTANS protein to promote flowering in response to day length. EMBO J. 2017;36(7):904-18.

98. Boden SA, Weiss D, Ross JJ, Davies NW, Trevaskis B, Chandler PM, Swain SM EARLY FLOWERING3 regulates FLOWERING in spring barley by mediating gibberellin production and FLOWERING LOCUS T expression. Plant Cell. 2014;26(4):1557-69.

99. Huang Z, Shi T, Zheng B, Yumul RE, Liu X, You C, Gao Z, Xiao L, Chen X. APETALA2 antagonizes the transcriptional activity of AGAMOUS in regulating floral stem cells in Arabidopsis thaliana. New Phytol. 2017;215(3): 1197-209.

100. Sayou C, Nanao MH, Jamin M, Posé D, Thévenon E, Grégoire L, Tichtinsky G, Denay G, Ott F, Llobet MP. A SAM oligomerization domain shapes the genomic binding landscape of the LEAFY transcription factor. Nat Commun 2016;7:11222.

101. Tang M, Tao YB, Fu Q, Song Y, Niu L, Xu ZF. An ortholog of LEAFY in Jatropha curcas regulates flowering time and floral organ development. Sci Rep. 2016:6(x):37306.

102. Chahtane H, Vachon G, Masson M, Thévenon E, Périgon S, Mihajlovic N, Kalinina A, Michard R, Moyroud E, Monniaux M. A variant of LEAFY reveals its capacity to stimulate meristem development by inducing RAX1. Plant J. 2013:74(4):678-89.

103. Wu M-F, Yamaguchi N, Xiao J, Bargmann B, Estelle M, Sang Y, Wagner D. Auxin-regulated chromatin switch directs acquisition of flower primordium founder fate. Elife. 2015;4:e09269.

104. Yamaguchi N, Wu M-F, Winter CM, Wagner D. LEAFY and polar auxin transport coordinately regulate Arabidopsis flower development. Plants. 2014;3(2):251-65.

105. Goslin K, Zheng B, Serrano-Mislata A, Rae L, Ryan PT, Kwaśniewska K, Thomson B, O'Maoileidigh D, Wellmer F, Graciet E. Transcription factor Interplay between LEAFY and APETALA1/CAULIFLOWER during Floral Initiation. Plant Physiol. 2017;174:00098.02017.

106. Grimplet J, Agudelo-Romero P, Teixeira RT, Martinez-Zapater JM, Fortes AM. Structural and functional analysis of the GRAS gene family in grapevine indicates a role of GRAS proteins in the control of development and stress responses. Front Plant Sci. 2016;7(e39547):353.

107. Hendelman A, Ran S, Zemach H, Arazi T. The tomato NAC transcription factor SINAM2 is involved in flower-boundary morphogenesis. J Exp Bot. 2013;64(18):5497-507.

108. Leydon AR, Beale KM, Woroniecka K, Castner E, Chen J, Horgan C, Palanivelu $\mathrm{R}$, Johnson MA. Three MYB transcription factors control pollen tube differentiation required for sperm release. Curr Biol. 2013;23(13):1209-14.

Ready to submit your research? Choose BMC and benefit from:

- fast, convenient online submission

- thorough peer review by experienced researchers in your field

- rapid publication on acceptance

- support for research data, including large and complex data types

- gold Open Access which fosters wider collaboration and increased citations

- maximum visibility for your research: over $100 \mathrm{M}$ website views per year

At $\mathrm{BMC}$, research is always in progress.

Learn more biomedcentral.com/submission 